\title{
ON THE LONG-TIME BEHAVIOUR OF A CLASS OF PARABOLIC SPDE'S: MONOTONICITY METHODS AND EXCHANGE OF STABILITY
}

\author{
Benjamin Bergé ${ }^{1}$ And Bruno Saussereau ${ }^{2}$
}

\begin{abstract}
In this article we prove new results concerning the structure and the stability properties of the global attractor associated with a class of nonlinear parabolic stochastic partial differential equations driven by a standard multidimensional Brownian motion. We first use monotonicity methods to prove that the random fields either stabilize exponentially rapidly with probability one around one of the two equilibrium states, or that they set out to oscillate between them. In the first case we can also compute exactly the corresponding Lyapunov exponents. The last case of our analysis reveals a phenomenon of exchange of stability between the two components of the global attractor. In order to prove this asymptotic property, we show an exponential decay estimate between the random field and its spatial average under an additional uniform ellipticity hypothesis.
\end{abstract}

Mathematics Subject Classification. 60H10, 60H15.

Received October 12, 2004. Revised February 15, 2005.

\section{INTRODUCTION AND PRELIMINARIES}

Consider a filtered probability space $\left(\Omega, \mathcal{F},\left(\mathcal{F}_{t}\right)_{t \in \mathbb{R}^{+}}, \mathbb{P}\right)$ on which is defined a standard $r$-dimensional Brownian motion $\left(W_{t}\right)_{t \in \mathbb{R}^{+}}=\left(W_{t}^{1}, \ldots, W_{t}^{r}\right)_{t \in \mathbb{R}^{+}}$. We denote by $D \subset \mathbb{R}^{d}$ a bounded connected open subset, satisfying the cone property and $\partial D$ is its boundary.

Our work is devoted to the long-time behaviour of the solution of the following class of parabolic SPDE's

$$
\left\{\begin{aligned}
\mathrm{d} u(x, t)= & (\operatorname{div}(k(x, t) \nabla u(x, t))+g(u(x, t))) \mathrm{d} t \\
& +\sum_{j=1}^{r} h_{j}(u(x, t)) \mathrm{d} W_{t}^{j}, \quad(x, t) \in D \times(0,+\infty), \\
u(x, 0)= & \varphi(x) \in\left(u_{0}, u_{1}\right), \quad x \in D, \\
\frac{\partial u(x, t)}{\partial n(k)}= & 0, \quad(x, t) \in \partial D \times[0,+\infty),
\end{aligned}\right.
$$

Keywords and phrases. Parabolic stochastic partial differential equations, asymptotic behaviour, monotonicity methods.

${ }^{1}$ Institut de Mathématiques, Université de Neuchâtel, Rue Émile Argand, 11, 2007 Neuchâtel, Switzerland; benjamin.berge@unine.ch

2 Département de Mathématiques, Université de Franche-Comté, 16 route de Gray, 25030 Besançon Cedex, France; bruno.saussereau@univ-fcomte.fr

(c) EDP Sciences, SMAI 2005 
where $\nabla$ denotes the gradient vector, $\frac{\partial}{\partial n(k)}$ is the conormal derivative relative to $k(\cdot, \cdot)$ and $u_{0}, u_{1}$ are reals such that $u_{0}<u_{1}$.

These kinds of SPDE's stand for a possible stochastic extension of deterministic phenomena: nerve pulse propagation, flame propagation or population dynamics (see Aronson and Weinberger [2]). For instance, the evolution of gene densities of a migrating species may be modelled by solution of (1.1). The second order differential operator enables us to take into account space- and time-dependant diffusions in the domain $D$ (see Murray [14]). Moreover, the drift term $g$ and the noise terms $h_{j}$ all vanish at $u_{0}=0$ and $u_{1}=1$. In the simplest and classical cases of logistic type nonlinearities, $g$ is of the form $g(u)=u(1-u)$. This leads to the KPP equation (see Kolmogorov et al. [12]), also studied in recent articles (see for instance Manthey and Mittmann [13], Øksendal et al. [15] and Øksendal et al. [16]). If we start from the constant initial condition $u_{0}$ (resp. $u_{1}$ ) then the random field $u_{\varphi}(x, t, \omega)=u_{0}$ (resp. $\left.u_{\varphi}(x, t, \omega)=u_{1}\right)$ solves the SPDE (1.1). So an interesting question is to ask if those two equilibrium states $u_{0}$ and $u_{1}$ are attractor or not.

In Chueshov and Vuillermot [6] and Chueshov and Vuillermot [7], the authors give a complete answer to this question when $r=1$ and the drift and the noise terms are proportional. If the stochastic integration is interpreted in the sense of Stratonovitch, they show that two behaviours are possible. First, one of the two steady states is globally asymptotically stable in probability and the second is unstable in probability. The second behaviour unveils a recurrence phenomenon. In Itô's case Chueshov and Vuillermot [7], they prove slightly different results if the drift term is larger than the noise: an exchange of stability between the two steady states appears. Bergé et al. [3] extend the results of Chueshov and Vuillermot [7] in a multidimensional white noise frame, and taking different drift and noise terms. The signs of the coefficients $g$ and $h$ remain constant but are not necessarily equal. In their work (as in Chueshov and Vuillermot [7]), the recurrence phenomenon does not appear. In this article, we improve all these results to the case of a general drift, especially not necessarily with constant sign. Of course, this allows us to write the SPDE (1.1) in the Stratonovitch or in the Itô sense. So in this work, the asymptotic behaviour exhibits all the possible behaviours encountered in the preceding articles. In Hetzer et al. [9], the authors study the asymptotic behaviour of positive solutions for stochastic parabolic equations of Fisher type which is also a generalization of results by Chueshov and Vuillermot [6]. We compare their results with ours in the last section.

Just below, we give the precise meaning of the SPDE's (1.1) and state our hypotheses. In Section 2 we recall the comparison principle with respect to the initial data which is the main tool of the first part devoted to the application of monotonicity methods for long-time behaviour. These methods are widely used in the theory of monotone random systems (see Hetzer et al. [5]). The alternative behaviours we met are essentially the same that the ones occuring in the theory of stochastic ordinary differential equations (see Sect. 6.6.2 of Hetzer et al. [5]). We prove that under a subordination condition on the drift term relative to the noise, the global attractor is exactly one of the two equilibrium states of (1.1) (see Th. 2.6 below). We also compute Lyapunov's exponents. Under another hypothesis, we prove that the random field is set out to oscillate between the two steady states in a recurrent way (see Th. 2.12). We stress that the technics involved in Section 2 are essentially the same that the ones used in Bergé et al. [3] and Chueshov and Vuillermot [7]. However, we synthetize and generalize the results of these works. We also explain the limits of these technics.

In the last possible case of hypotheses on $g$ and $h_{j}$ 's, the monotonicity methods fail. We overcome this difficulty in Section 3 using a comparison between the random field and its spatial average thanks to an exponential decay estimate. Actually, under an additional hypothesis on the ellipticity constant of the divergence operator, we focus on the spatial average of the random field. The long-time behaviour of the spatial average process is obtained thanks to similar methods encountered in classical results on stochastic differential equations (in short SDE's). Finally, we construct a Bernoulli random variable with values in the set of the steady states of the problem (1.1), which is the global attractor of the solution of the SPDE (see Th. 3.5).

Our main results are stated in Theorems 2.6, 2.12 and 3.5. They improve and partially include those of Bergé et al. [3], Chueshov and Vuillermot [6] and Chueshov and Vuillermot [7]. Section 4 is devoted to the proofs of auxiliary results stated in Section 3.

We give concluding remarks in Section 5 and focus on comparisons with previous works. 
Now we state our hypotheses and give the notion of solution we choose.

(K) The symmetric matrix-valued function $k$ is such that there exist three positive constants $k_{1}, k_{2}$ and $C$ such that, for all $q \in \mathbb{R}^{d}$ and all $(x, t) \in D \times \mathbb{R}^{+}$,

$$
k_{1}|q|^{2} \leqslant\langle k(x, t) q, q\rangle_{\mathbb{R}^{d}} \leqslant k_{2}|q|^{2}
$$

where $\langle\cdot, \cdot\rangle_{\mathbb{R}^{d}}$ denotes the canonical inner product of $\mathbb{R}^{d}$, with the associated norm $|\cdot|$. In addition, for all $i, j \in\{1, \ldots, d\}$ and all $s, t \in \mathbb{R}^{+}$, we have

$$
\sup _{x \in D}\left|k_{i, j}(x, t)-k_{i, j}(x, s)\right| \leqslant C|t-s| .
$$

(G) The function $g$ belongs to $\mathcal{C}^{2}\left(\left[u_{0}, u_{1}\right], \mathbb{R}\right)$ and $g\left(u_{0}\right)=g\left(u_{1}\right)=0$.

(H) The functions $h_{j}$ belong to $\mathcal{C}^{2}\left(\left[u_{0}, u_{1}\right], \mathbb{R}\right)$. We note $h=\left(h_{1}, \ldots, h_{r}\right)$ and we assume that $h\left(u_{0}\right)=$ $h\left(u_{1}\right)=0$. In addition, we assume that $|h(u)|>0$ on the open set $\left(u_{0}, u_{1}\right)$, and that $\left|h^{\prime}\left(u_{0}\right)\right|>0$ and $\left|h^{\prime}\left(u_{1}\right)\right|>0$.

(I) The initial condition is non-random and satisfies

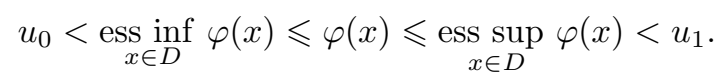

We write $\|\cdot\|_{2}$ for the usual $L^{2}(D)$-norm and $\mathcal{C}\left((0, T) ; L^{2}(D)\right)$ for the space of all continuous mappings from the interval $(0, T)$ into $L^{2}(D)$ when $T \in \mathbb{R}^{+}$. Among all the possible ways to define a notion of solution to Problem (1.1) (see Sanz-Solé and Vuillermot [17]) we choose the following.

Definition 1.1. We say that the $L^{2}(D)$-valued, measurable random field $\left(u_{\varphi}(\cdot, t)\right)_{t \in \mathbb{R}^{+}}$defined on $(\Omega, \mathcal{F}$, $\left.\left(\mathcal{F}_{t}\right)_{t \in \mathbb{R}^{+}}, \mathbb{P}\right)$ is a solution-random field to Problem (1.1) if the following conditions hold:

(1) $\left(u_{\varphi}(\cdot, t)\right)_{t \in \mathbb{R}^{+}}$is adapted to the filtration $\left(\mathcal{F}_{t}\right)_{t \in \mathbb{R}^{+}}$;

(2) for every $T \in \mathbb{R}^{+}$we have $u_{\varphi} \in L^{2}\left((0, T) \times \Omega ; H^{1}(D)\right) \cap L^{2}\left(\Omega ; \mathcal{C}\left((0, T) ; L^{2}(D)\right)\right)$ and consequently

$$
\mathbb{E} \int_{0}^{T}\left\|u_{\varphi}(\cdot, s)\right\|_{2}^{2}+\left\|\nabla u_{\varphi}(\cdot, s)\right\|_{2}^{2} \mathrm{~d} s<+\infty
$$

(3) we have $u_{\varphi}(x, t) \in\left(u_{0}, u_{1}\right)(\mathrm{d} x \otimes \mathbb{P})$-a.e. for every $t \in \mathbb{R}^{+}$and, for every $T \in \mathbb{R}^{+}$, the relation

$$
\begin{aligned}
\int_{D} v(x) u_{\varphi}(x, t) \mathrm{d} x= & \int_{D} v(x) \varphi(x) \mathrm{d} x \\
& -\int_{D} \int_{0}^{t}\left\langle\nabla v(x), k(x, s) \nabla u_{\varphi}(x, s)\right\rangle_{\mathbb{R}^{d}} \mathrm{~d} s \mathrm{~d} x+\int_{D} v(x) \int_{0}^{t} g\left(u_{\varphi}(x, s)\right) \mathrm{d} s \mathrm{~d} x \\
& +\sum_{j=1}^{r} \int_{D} v(x) \int_{0}^{t} h_{j}\left(u_{\varphi}(x, s)\right) \mathrm{d} W_{s}^{j} \mathrm{~d} x
\end{aligned}
$$

holds $\mathbb{P}$-a.e. for every $v \in H^{1}(D)$ and every $t \in[0, T]$.

According to the results of Bergé et al. [3], there is a unique solution $\left(u_{\varphi}(\cdot, t)\right)_{t \in \mathbb{R}^{+}}$of $(1.1)$.

\section{Monotonicity Methods For Stability And LyApunov EXPONENTS}

\subsection{Generalities on the asymptotic behaviour}

The monotonicity methods we will develop below are based on the following comparison principle related to the initial data (see Bergé et al. [3]). 
Theorem 2.1. Let $\psi_{1}$ and $\psi_{2}$ be two functions satisfying Hypothesis $(I)$. Let $\left(u_{\psi_{1}}(\cdot, t)\right)_{t \in \mathbb{R}^{+}}\left(r e s p .\left(u_{\psi_{2}}(\cdot, t)\right)_{t \in \mathbb{R}^{+}}\right)$ be the solution of the SPDE (1.1) with initial condition $\psi_{1}$ (resp. $\left.\psi_{2}\right)$. In addition, we assume that

$$
\psi_{1}(x) \leqslant \psi_{2}(x)
$$

$\mathrm{d} x$-a.e. Then we have

$\mathrm{d} x \otimes \mathbb{P}$-a.e. for all $t \in \mathbb{R}^{+}$.

$$
u_{\psi_{1}}(x, t) \leqslant u_{\psi_{2}}(x, t)
$$

The key point is to find initial conditions $\psi$ for which the investigation of the random field $\left(u_{\psi}(\cdot, t)\right)_{t \in \mathbb{R}^{+}}$ is simpler than $\left(u_{\varphi}(\cdot, t)\right)_{t \in \mathbb{R}^{+}}$. The simplest ones are those starting from a constant initial condition. That is the reason why we apply the comparison principle with constant initial conditions $\varphi_{1}=\underset{x \in D}{\operatorname{ess} \inf } \varphi(x)$ and $\varphi_{2}=\operatorname{ess} \sup \varphi(x)$. Obviously, the corresponding random fields solution of the SPDE's (1.1) are in fact solution of the SDE's

$$
\begin{gathered}
v_{1}(t)=\varphi_{1}+\int_{0}^{t} g\left(v_{1}(s)\right) \mathrm{d} s+\sum_{j=1}^{r} \int_{0}^{t} h_{j}\left(v_{1}(s)\right) \mathrm{d} W_{s}^{j}, \\
v_{2}(t)=\varphi_{2}+\int_{0}^{t} g\left(v_{2}(s)\right) \mathrm{d} s+\sum_{j=1}^{r} \int_{0}^{t} h_{j}\left(v_{2}(s)\right) \mathrm{d} W_{s}^{j}
\end{gathered}
$$

for $t \geqslant 0$. We deduce from the comparison theorem that

$$
u_{0} \leqslant v_{1}(t) \leqslant u_{\varphi}(x, t) \leqslant v_{2}(t) \leqslant u_{1}
$$

$\mathrm{d} x \otimes \mathbb{P}$-a.e. for all $t \in \mathbb{R}^{+}$.

We first state that equilibrium points $u_{0}$ and $u_{1}$ are non attainable in finite time.

Proposition 2.2. We suppose $(K),(G),(H)$ and $(I)$. Then we have

$$
\mathbb{P}\left\{\exists t>0,\left\|u_{\varphi}(\cdot, t)-u_{j}\right\|_{\infty}=0\right\}=0
$$

for $j=0,1$.

Proof. We start by proving the result for $j=0$. Thanks to the inequalities (2.3) it is sufficient to prove the result for the process $\left(v_{1}(t)\right)_{t \in \mathbb{R}^{+}}$. Let $\varepsilon$ be in $(0,1)$ and $\left(r_{n}\right)_{n \in \mathbb{N}}$ be a sequence of radii decreasing to 0 . For each $n \in \mathbb{N}, f_{n}$ is a twice-differentiable function on $\left[u_{0}, u_{1}\right]$ such that $f_{n}(u)=\left(u-u_{0}\right)^{-\varepsilon}$ if $u \geqslant u_{0}+r_{n}$.

If $\varphi_{1}>u_{0}+r_{n}$, we define the sequence of increasing stopping times $T_{n}=\inf \left\{t>0:\left|v_{1}(t)-u_{0}\right|=r_{n}\right\}$.

Now we apply Itô's formula to $\mathrm{e}^{-\beta t} f_{n}$ between 0 and $t \wedge T_{n}, \beta$ to be fixed later. We obtain

$$
\begin{aligned}
\mathrm{e}^{-\beta\left(t \wedge T_{n}\right)} f_{n}\left(v_{1}\left(t \wedge T_{n}\right)\right)= & f_{n}\left(\varphi_{1}\right)-\int_{0}^{t \wedge T_{n}} \beta \mathrm{e}^{-\beta s} f_{n}\left(v_{1}(s)\right) \mathrm{d} s+\int_{0}^{t \wedge T_{n}} \mathrm{e}^{-\beta s} f_{n}^{\prime}\left(v_{1}(s)\right) g\left(v_{1}(s)\right) \mathrm{d} s \\
& +\int_{0}^{t \wedge T_{n}} \frac{1}{2} \mathrm{e}^{-\beta s} f_{n}^{\prime \prime}\left(v_{1}(s)\right)\left|h\left(v_{1}(s)\right)\right|^{2} \mathrm{~d} s+\sum_{j=1}^{r} \int_{0}^{t \wedge T_{n}} \mathrm{e}^{-\beta s} f_{n}^{\prime}\left(v_{1}(s)\right) h_{j}\left(v_{1}(s)\right) \mathrm{d} W_{s}^{j}
\end{aligned}
$$

Taking expectation, this leads to

$$
\begin{aligned}
\mathbb{E}\left[\mathrm{e}^{-\beta\left(t \wedge T_{n}\right)} f_{n}\left(v_{1}\left(t \wedge T_{n}\right)\right)\right] & =f_{n}\left(\varphi_{1}\right) \\
+ & \mathbb{E}\left[\int_{0}^{t \wedge T_{n}} \mathrm{e}^{-\beta s}\left(f_{n}^{\prime}\left(v_{1}(s)\right) g\left(v_{1}(s)\right)+\frac{1}{2} f_{n}^{\prime \prime}\left(v_{1}(s)\right)\left|h\left(v_{1}(s)\right)\right|^{2}-\beta f_{n}\left(v_{1}(s)\right)\right) \mathrm{d} s\right] .
\end{aligned}
$$


But for $s \in\left[0, t \wedge T_{n}\right]$ we have

$$
\begin{aligned}
f_{n}^{\prime}\left(v_{1}(s)\right) g\left(v_{1}(s)\right)+\frac{1}{2} f_{n}^{\prime \prime}\left(v_{1}(s)\right)\left|h\left(v_{1}(s)\right)\right|^{2}= & -\varepsilon\left(v_{1}(s)-u_{0}\right)^{-\varepsilon-1} g\left(v_{1}(s)\right) \\
& +\frac{1}{2} \varepsilon(1+\varepsilon)\left(v_{1}(s)-u_{0}\right)^{-2-\varepsilon}\left|h\left(v_{1}(s)\right)\right|^{2} \\
= & \varepsilon\left(v_{1}(s)-u_{0}\right)^{-\varepsilon}\left[-\frac{g\left(v_{1}(s)\right)}{v_{1}(s)-u_{0}}+\frac{1+\varepsilon}{2} \frac{\left|h\left(v_{1}(s)\right)\right|^{2}}{\left(v_{1}(s)-u_{0}\right)^{2}}\right] \\
\leqslant & K\left(v_{1}(s)-u_{0}\right)^{-\varepsilon},
\end{aligned}
$$

where $K$ is a bound of the function

$$
u \mapsto-\frac{g(u)}{u-u_{0}}+\frac{1+\varepsilon}{2} \frac{|h(u)|^{2}}{\left(u-u_{0}\right)^{2}}
$$

Therefore, if we choose $\beta>K$, and using (2.5), the relation (2.4) becomes

$$
\mathbb{E}\left[\mathrm{e}^{-\beta\left(t \wedge T_{n}\right)} f_{n}\left(v_{1}\left(t \wedge T_{n}\right)\right)\right] \leqslant f_{n}\left(\varphi_{1}\right)
$$

Since

$$
\mathrm{e}^{-\beta\left(t \wedge T_{n}\right)} f_{n}\left(v_{1}\left(t \wedge T_{n}\right)\right) \mathbb{1}_{\left\{T_{n}<+\infty\right\}} \underset{t \rightarrow+\infty}{\longrightarrow} \mathrm{e}^{-\beta T_{n}} f_{n}\left(v_{1}\left(T_{n}\right)\right) \mathbb{1}_{\left\{T_{n}<+\infty\right\}}=\mathrm{e}^{-\beta T_{n}} r_{n}^{-\varepsilon} \mathbb{1}_{\left\{T_{n}<+\infty\right\}},
$$

Fatou's lemma implies

$$
\mathbb{E}\left[\mathrm{e}^{-\beta T_{n}} \mathbb{1}_{\left\{T_{n}<+\infty\right\}}\right] \leqslant\left(\varphi_{1}-u_{0}\right)^{-\varepsilon} r_{n}^{\varepsilon}
$$

where we have used $v_{1}\left(T_{n}\right)=r_{n}+u_{0}$. Defining $T=\lim _{n \rightarrow+\infty} T_{n}$ (P्P-a.s.) and letting $n$ tend to infinity in (2.6), we have by the monotone convergence theorem,

$$
\mathbb{E}\left[\mathrm{e}^{-\beta T} \mathbb{1}_{\{T<+\infty\}}\right]=0 .
$$

Consequently, $\mathbb{P}\{T=+\infty\}=1$ and the result follows.

The proof of the result for $j=1$ is omitted. We use the same arguments with the random process $\left(v_{2}(t)\right)_{t \in \mathbb{R}^{+}}$ and a suitable sequence of functions $\left(f_{n}\right)_{n \in \mathbb{N}}$.

In the above proof, $\left(v_{1}(t)\right)_{t \in \mathbb{R}^{+}}$and $\left(v_{2}(t)\right)_{t \in \mathbb{R}^{+}}$, which solves respectively the $\operatorname{SDE}(2.1)$ and (2.2), play an important role that will increase in the following. We recall that the Feller function is a useful tool used to investigate the long-time behaviour of SDE's. This function is defined as a solution of the ordinary differential equation

$$
\frac{1}{2}|h(u)|^{2} F^{\prime \prime}(u)+g(u) F^{\prime}(u)=0,
$$

for $u \in\left(u_{0}, u_{1}\right)$. We have to study the local behaviour of $F$ near $u_{0}$ and $u_{1}$ (see Ikeda and Watanabe [11], p. 362). So we write that

$$
F(u)=\int_{\nu}^{u} \exp \left(-2 \int_{\mu}^{y} \frac{g(z)}{|h(z)|^{2}} \mathrm{~d} z\right) \mathrm{d} y
$$

where $\mu$ and $\nu$ are two arbitrary points of $\left(u_{0}, u_{1}\right)$. 
We precise the behaviour of the Feller function around $u_{0}$ and $u_{1}$ in the next proposition.

Proposition 2.3. For the function $F$, we have those four following cases.

(A) If $2 g^{\prime}\left(u_{0}\right)<\left|h^{\prime}\left(u_{0}\right)\right|^{2}$ and if $2 g^{\prime}\left(u_{1}\right) \geqslant\left|h^{\prime}\left(u_{1}\right)\right|^{2}$ then

$$
\lim _{u \rightarrow u_{0}} F(u)>-\infty \text { and } \lim _{u \rightarrow u_{1}} F(u)=+\infty
$$

(B) If $2 g^{\prime}\left(u_{0}\right) \geqslant\left|h^{\prime}\left(u_{0}\right)\right|^{2}$ and if $2 g^{\prime}\left(u_{1}\right)<\left|h^{\prime}\left(u_{1}\right)\right|^{2}$, then

$$
\lim _{u \rightarrow u_{0}} F(u)=-\infty \text { and } \lim _{u \rightarrow u_{1}} F(u)<+\infty .
$$

(C) If $2 g^{\prime}\left(u_{0}\right) \geqslant\left|h^{\prime}\left(u_{0}\right)\right|^{2}$ and if $2 g^{\prime}\left(u_{1}\right) \geqslant\left|h^{\prime}\left(u_{1}\right)\right|^{2}$, then

$$
\lim _{u \rightarrow u_{0}} F(u)=-\infty \text { and } \lim _{u \rightarrow u_{1}} F(u)=+\infty .
$$

(D) If $2 g^{\prime}\left(u_{0}\right)<\left|h^{\prime}\left(u_{0}\right)\right|^{2}$ and if $2 g^{\prime}\left(u_{1}\right)<\left|h^{\prime}\left(u_{1}\right)\right|^{2}$, then

$$
\lim _{u \rightarrow u_{0}} F(u)>-\infty \text { and } \lim _{u \rightarrow u_{1}} F(u)<+\infty .
$$

Remark 2.4. It is worth noting that, since the function $g$ may vanish, these four cases are possible. In Chueshov and Vuillermot [6], the case (C) may occur, but not the case (D) whereas in Bergé et al. [3] and in Chueshov and Vuillermot [7], the case (D) appears but not the case (C). This shows that our framework encompasses all the cases of Bergé et al. [3], Chueshov and Vuillermot [6] and Chueshov and Vuillermot [7].

Proof. We essentially use the same arguments as in Bergé et al. [3]. It is easy to check that there exist $c_{1}$ and $c_{2}$ such that for $u \in\left(u_{0}, u_{1}\right)$,

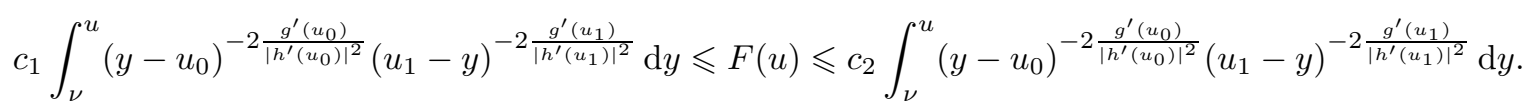

The behaviour of $F$ around $u_{0}$ and $u_{1}$ is then driven by the integrability of the function

$$
y \mapsto\left(y-u_{0}\right)^{-2 \frac{g^{\prime}\left(u_{0}\right)}{\left|h^{\prime}\left(u_{0}\right)\right|^{2}}}
$$

around $u_{0}$ and by the integrability of the function

$$
y \mapsto\left(u_{1}-y\right)^{-2 \frac{g^{\prime}\left(u_{1}\right)}{\left|h^{\prime}\left(u_{1}\right)\right|^{2}}}
$$

around $u_{1}$. This implies cases (A), (B), (C) and (D).

We begin with the simplest cases (A) and (B), where the global attractor is exactly one of the two equilibrium states.

\subsection{Global asymptotic stability: the cases (A) and (B)}

In cases $(\mathrm{A})$ and $(\mathrm{B})$, we investigate in details the long-time behaviour of the random field $\left(u_{\varphi}(\cdot, t)\right)_{t \in \mathbb{R}^{+}}$and are able to compute the Lyapunov exponents.

Let $A$ be the function defined for $u \in\left(u_{0}, u_{1}\right)$ by

$$
A(u)=\int_{\mu}^{u} \frac{\mathrm{d} z}{\left(z-u_{0}\right)\left(u_{1}-z\right)}
$$


The function $A$ is useful in the computation of Lyapunov's exponents. The straightforward following lemma shows that the function $A$ diverges logarithmically around $u_{0}$ and $u_{1}$.

Lemma 2.5. Let $c \in\left(0, u_{1}-u_{0}\right)$ be a real. Then

(i) there exist two constants $c_{1}$ and $c_{2}$ such that, for all $y \in\left(u_{0}, u_{1}-c\right)$,

$$
c_{1}+A(y) \leqslant \frac{1}{u_{1}-u_{0}} \ln \left(y-u_{0}\right) \leqslant c_{2}+A(y) ;
$$

(ii) there exist two constants $c_{3}$ and $c_{4}$ such that, for all $y \in\left(u_{0}+c, u_{1}\right)$,

$$
c_{3}+A(y) \leqslant \frac{1}{u_{1}-u_{0}} \ln \left(u_{1}-y\right) \leqslant c_{4}+A(y) .
$$

The following theorem states that the global attractor is non random and consists of the two stationary states $u_{0}$ and $u_{1}$. It is worth noting that in the cases (A) and (B), the roles of $u_{0}$ and $u_{1}$ are inverted. In both cases, we can exactly determine the corresponding Lyapunov exponents. This result is a direct consequence of the comparison Theorem.

Theorem 2.6. In the case (A), the following relation holds

$$
\mathbb{P}\left\{\lim _{t \rightarrow+\infty}\left\|u_{\varphi}(\cdot, t)-u_{0}\right\|_{\infty}=0\right\}=1
$$

and we explicitly compute the Lyapunov exponent

$$
\lim _{t \rightarrow+\infty} \frac{1}{t} \ln \left\|u_{\varphi}(\cdot, t)-u_{0}\right\|_{\infty}=g^{\prime}\left(u_{0}\right)-\frac{1}{2}\left|h^{\prime}\left(u_{0}\right)\right|^{2}<0 \mathbb{P} \text {-a.s. }
$$

In the case (B), the following relation holds

$$
\mathbb{P}\left\{\lim _{t \rightarrow+\infty}\left\|u_{\varphi}(\cdot, t)-u_{1}\right\|_{\infty}=0\right\}=1
$$

and we explicitly compute the Lyapunov exponent

$$
\lim _{t \rightarrow+\infty} \frac{1}{t} \ln \left\|u_{\varphi}(\cdot, t)-u_{1}\right\|_{\infty}=g^{\prime}\left(u_{1}\right)-\frac{1}{2}\left|h^{\prime}\left(u_{1}\right)\right|^{2}<0 \mathbb{P} \text {-a.s. }
$$

Proof. We only prove the case (A) (the proof of (B) is similar and omitted). We deduce from (2.3) that

$$
\mathbb{P}\left\{\lim _{t \rightarrow+\infty}\left|v_{2}(t)-u_{0}\right|=0\right\} \leqslant \mathbb{P}\left\{\lim _{t \rightarrow+\infty}\left\|u_{\varphi}(\cdot, t)-u_{0}\right\|_{\infty}=0\right\} .
$$

A direct application of classical results on the asymptotic behaviour of SDE's (see Ikeda and Watanabe [11], Th. 3.1, p. 362) and Proposition 2.3 yield

$$
\mathbb{P}\left\{\lim _{t \rightarrow+\infty} v_{2}(t)=u_{0}\right\}=\lim _{\substack{b \uparrow u_{1} \\ a \downarrow u_{0}}} \frac{F(b)-F\left(\varphi_{2}\right)}{F(b)-F(a)}=1
$$

hence the relation (2.9) holds. The further argument is the same as in Bergé et al. [3]. 
In order to give more precise properties of the two steady states, we recall three definitions related to stability in a random framework. Analogous notions of stability for ordinary stochastic differential equations are used to study random dynamical systems generated by ordinary Itô equations (Arnold [1], Gihman and Skorohod [8], Hasminskii [10]).

Definition 2.7. We say that $u_{0,1} \in\left\{u_{0}, u_{1}\right\}$ is stable in probability if the relation

$$
\lim _{\left\|\varphi-u_{0,1}\right\|_{\infty} \rightarrow 0} \mathbb{P}\left\{\sup _{t \in \mathbb{R}^{+}}\left\|u_{\varphi}(\cdot, t)-u_{0,1}\right\|_{\infty}>\varepsilon\right\}=0
$$

holds for every $\varepsilon>0$.

Definition 2.8. We say that $u_{0,1} \in\left\{u_{0}, u_{1}\right\}$ is globally asymptotically stable in probability if relation (2.13) holds and if we have

$$
\mathbb{P}\left\{\lim _{t \rightarrow+\infty}\left\|u_{\varphi}(\cdot, t)-u_{0,1}\right\|_{\infty}=0\right\}=1
$$

for every initial condition $\varphi$ satisfying hypothesis (I).

Definition 2.9. We say that $u_{0,1}$ is unstable in probability if relation (2.13) does not hold.

To achieve our study of the asymptotic behaviour of the process $\left(u_{\varphi}(\cdot, t)\right)_{t \in \mathbb{R}^{+}}$in the cases $(\mathrm{A})$ and $(\mathrm{B})$, we give the stability properties of the equilibrium states.

Theorem 2.10. Assume that $(K),(G),(H)$ and $(I)$ hold. In the case $(A), u_{0}$ is globally asymptotically stable in probability and $u_{1}$ is unstable in probability.

In the case $(B), u_{1}$ is globally asymptotically stable in probability and $u_{0}$ is unstable in probability.

Remark 2.11. Intuitively the theorem claims that in the case $(\mathrm{A}), u_{0}$ attracts the random field whereas $u_{1}$ repels it. In the case (B), the roles of $u_{0}$ and $u_{1}$ are inverted.

Proof. In the case (A), we first prove that for all $\varepsilon>0$, and all $\delta_{1}>0$, there exists $\delta_{2} \in\left(0, \delta_{1}\right)$ such that for all $\varphi$ satisfying $\left\|\varphi-u_{0}\right\|_{\infty} \leqslant \delta_{2}$, then

$$
\mathbb{P}\left\{\left\|u_{\varphi}(\cdot, t)-u_{0}\right\|_{\infty}>\delta_{1} \text { for some } t>0\right\}<\varepsilon .
$$

Actually, as we have

$$
\mathbb{P}\left\{\left\|u_{\varphi}(\cdot, t)-u_{0}\right\|_{\infty}>\delta_{1} \text { for some } t>0\right\} \leqslant \mathbb{P}\left\{\left|v_{2}(t)-u_{0}\right|>\delta_{1} \text { for some } t>0\right\}<\varepsilon,
$$

it suffices to prove the second inequality. Let $F_{0}$ be the solution of the differential equation

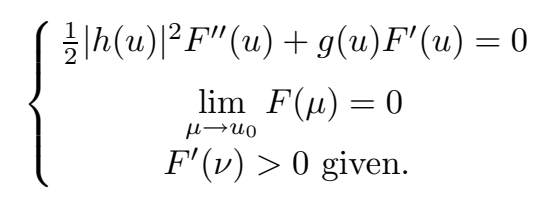

The hypothesis $2 g^{\prime}\left(u_{0}\right)<\left|h^{\prime}\left(u_{0}\right)\right|^{2}$ ensures that $F\left(u_{0}\right)$ exists (we take $\mu=u_{0}$ in formula $(2.8)$ ). As $F_{0}$ is strictly increasing, we have $F_{0}(u)>0$ for all $u \in\left(u_{0}, u_{1}\right)$. Let $\tau$ be the stopping time defined by $\tau=\inf \left\{t>0: v_{2}(t)>\right.$ $\left.\delta_{1}+u_{0}\right\}$. Applying Itô's formula between 0 and $\tau \wedge n$ for all $n \in \mathbb{N}$, we have

$$
\begin{aligned}
F_{0}\left(v_{2}(\tau \wedge n)\right)= & F_{0}\left(\varphi_{2}\right)+\int_{0}^{\tau \wedge n} \frac{1}{2}\left|h\left(v_{2}(s)\right)\right|^{2} F_{0}^{\prime \prime}\left(v_{2}(s)\right)+g\left(v_{2}(s)\right) F_{0}^{\prime}\left(v_{2}(s)\right) \mathrm{d} s \\
& +\sum_{j=1}^{r} \int_{0}^{\tau \wedge n} h_{j}\left(v_{2}(s)\right) F^{\prime}\left(v_{2}(s)\right) \mathrm{d} W_{s}^{j} .
\end{aligned}
$$


Since $F_{0}$ satisfies relations $(2.15)$ and $\varphi_{2}>u_{0}$, taking expectation yields

$$
\mathbb{E}\left[F_{0}\left(v_{2}(\tau \wedge n)\right) \mathbb{1}_{\{\tau<+\infty\}}\right] \leqslant \mathbb{E} F_{0}\left(v_{2}(\tau \wedge n)\right)=\mathbb{E} F_{0}\left(\varphi_{2}\right)=F_{0}\left(\varphi_{2}\right)>0 .
$$

Applying Fatou's lemma to (2.16) we find

$$
\mathbb{E}\left[F_{0}\left(v_{2}(\tau)\right) \mathbb{1}_{\{\tau<+\infty\}}\right] \leqslant F_{0}\left(\varphi_{2}\right) .
$$

Since $F_{0}\left(v_{2}(\tau)\right) \mathbb{1}_{\{\tau<+\infty\}}=F_{0}\left(\delta_{1}\right)$, we have

$$
\mathbb{P}\{\tau<+\infty\} \leqslant \frac{F_{0}\left(\varphi_{2}\right)}{F_{0}\left(\delta_{1}\right)} \leqslant \frac{F_{0}\left(\delta_{2}\right)}{F_{0}\left(\delta_{1}\right)} .
$$

We choose $\delta_{2}$ such that $F_{0}\left(\delta_{2}\right)=\varepsilon F_{0}\left(\delta_{1}\right)$. This choice is possible because the function $F_{0}$ is strictly increasing, $F_{0}\left(u_{0}\right)=0$ and $F_{0}(u)$ tends to $\infty$ when $u$ tends to $u_{1}$. With this choice for $\delta_{2}$ in (2.17), we obtain $\mathbb{P}\{\tau<$ $+\infty\} \leqslant \varepsilon$. Since $\{\tau<+\infty\}=\left\{\exists t>0, v_{2}(t) \geqslant \delta_{1}\right\}$ we have the result.

To the end, it remains to prove the unstability of the state $u_{1}$ in the case (A). Actually, we prove the following stronger result:

Thanks to the inequalities (2.3), we deduce that

$$
\forall \varepsilon>0 \quad \lim _{\left\|\varphi-u_{1}\right\|_{\infty} \rightarrow 0} \mathbb{P}\left\{\sup _{t \in \mathbb{R}^{+}}\left\|u_{\varphi}(\cdot, t)-u_{1}\right\|_{\infty} \geqslant \varepsilon\right\}=1 .
$$

$$
\mathbb{P}\left\{\sup _{t \in \mathbb{R}^{+}}\left|u_{1}-v_{2}(t)\right| \geqslant \varepsilon\right\} \leqslant \mathbb{P}\left\{\sup _{t \in \mathbb{R}^{+}}\left\|u_{\varphi}(\cdot, t)-u_{1}\right\|_{\infty} \geqslant \varepsilon\right\},
$$

so it remains to prove that

$$
\lim _{\left|\varphi_{2}-u_{1}\right| \rightarrow 0} \mathbb{P}\left\{\sup _{t \in \mathbb{R}^{+}}\left\|v_{2}(t)-u_{1}\right\|_{\infty} \geqslant \varepsilon\right\}=1 .
$$

Let $\varepsilon>0$ be fixed and $b$ a real such that $u_{1}-\varepsilon \leqslant \varphi_{2} \leqslant b<u_{1}$. We note $\tau_{u_{1}-\varepsilon, b}=\inf \left\{t \in \mathbb{R}^{+}: v_{2}(t) \notin\left(u_{1}-\varepsilon, b\right)\right\}$ the first exit time of the interval $\left(u_{1}-\varepsilon, b\right)$. We have

$$
\begin{aligned}
\mathbb{P}\left\{\sup _{t \in \mathbb{R}^{+}}\left|u_{1}-v_{2}(t)\right| \geqslant \varepsilon\right\} & \geqslant \mathbb{P}\left\{v_{2}\left(\tau_{u_{1}-\varepsilon, b}\right)=u_{1}-\varepsilon\right\} \\
& \geqslant \frac{F(b)-F\left(\varphi_{2}\right)}{F(b)-F\left(u_{1}-\varepsilon\right)},
\end{aligned}
$$

where the last inequality comes from classical arguments Ikeda and Watanabe [11]. The relation (2.18) is a direct consequence of letting $b$ tends to $u_{1}$ in the relation (2.19) combining with the result of Proposition 2.3.

To prove the stability of $u_{1}$ for the case (B), we have to show that for all $\varepsilon>0$, for all $0<\delta_{1}<u_{1}-u_{0}$ there exists $\delta_{2} \in\left(0, \delta_{1}\right)$ such that if $u_{1}-\delta_{2} \leqslant \varphi_{1}<u_{1}$, we have

$$
\mathbb{P}\left\{\exists t>0, v_{1}(t)<u_{1}-\delta_{1}\right\}<\varepsilon .
$$

The proof is analogous. Instead of the function $F_{0}$, we work with $F_{1}$, solution of the problem

$$
\left\{\begin{array}{c}
\frac{1}{2}|h(u)|^{2} F^{\prime \prime}(u)+g(u) F^{\prime}(u)=0 \\
\lim _{\mu \rightarrow u_{1}} F(\mu)=0 \\
F^{\prime}(\nu)<0 \text { given }
\end{array}\right.
$$

and note that $F_{1}$ is non-positive, increasing, and $F_{1}(u)$ tends to $-\infty$ when $u$ tends to $u_{0}$. 


\subsection{Recurrence properties: the case (C)}

In this section, we deal with the case $(\mathrm{C})$. This situation is more chaotic and an oscillation phenomenon sets in for large times. Indeed, we will see in the following theorem that $u_{\varphi}$ travels back and forth between the two stationary states $u_{0}$ and $u_{1}$ in a recurrent way in the sense that it can reach every point within $\left(u_{0}, u_{1}\right)$ almost surely in finite time.

Theorem 2.12. In the case $(C)$, the following assertions are true:

(i) $\limsup _{t \rightarrow+\infty} \operatorname{ess}_{x \in D} u_{\varphi}(x, t)=\limsup _{t \rightarrow+\infty} \operatorname{ess}_{x \in D} \inf _{\varphi}(x, t)=u_{1} \mathbb{P}$-a.s.;

(ii) $\liminf _{t \rightarrow+\infty} \operatorname{ess} \sup _{x \in D} u_{\varphi}(x, t)=\liminf _{t \rightarrow+\infty} \underset{x \in D}{\operatorname{ess} \inf } u_{\varphi}(x, t)=u_{0} \mathbb{P}-$ a.s.

Proof. Using the relations (2.3), we obtain

$$
u_{0} \leqslant \limsup _{t \rightarrow+\infty} v_{1}(t) \leqslant \limsup _{t \rightarrow+\infty} \operatorname{ess}_{x \in D} \inf _{x \in D} u_{\varphi}(x, t) \leqslant \limsup _{t \rightarrow+\infty} \operatorname{ess}_{x \in D} \sup _{\varphi} u_{\varphi}(x, t) \leqslant \limsup _{t \rightarrow+\infty} v_{2}(t) \leqslant u_{1}
$$

$\mathrm{d} x \otimes \mathbb{P}$-a.e. and for all $t \in \mathbb{R}^{+}$. To prove (i), it remains to show that $\limsup _{t \rightarrow+\infty} v_{1}(t)=u_{1} \mathbb{P}$-a.s., but this is a direct consequence of Ikeda and Watanabe [11], p. 362. Since

$$
u_{0} \leqslant \liminf _{t \rightarrow+\infty} v_{1}(t) \leqslant \liminf _{t \rightarrow+\infty} \operatorname{ess} \inf _{x \in D} u_{\varphi}(x, t) \liminf _{t \rightarrow+\infty} \operatorname{ess~sup}_{x \in D} u_{\varphi}(x, t) \leqslant \liminf _{t \rightarrow+\infty} v_{2}(t) \leqslant u_{1}
$$

$\mathrm{d} x \otimes \mathbb{P}$-a.e., the proof of (ii) is similar.

Corollary 2.13. Let $\mathcal{L}$ be a linear, positive, continuous functional on $L^{2}(D)$ such that $\mathcal{L}\left(\mathbb{1}_{D}\right)=1$, and let

$$
\tau_{y}=\inf \left\{t>0: \mathcal{L}\left(u_{\varphi}(\cdot, t)\right)=y\right\}
$$

be the first time that the process $\left(\mathcal{L}\left(u_{\varphi}(\cdot, t)\right)\right)_{t \in \mathbb{R}^{+}}$reaches $y$ (with convention $\left.\inf \emptyset=+\infty\right)$. Then, for all $y \in\left(u_{0}, u_{1}\right)$ we have

$$
\mathbb{P}\left\{\tau_{y}<+\infty\right\}=1
$$

Remark 2.14. An interesting application of this corollary is the choice of $\mathcal{L}$ as an average operator over any small ball strictly included in $D$. Let $x_{0} \in D$ and $\varepsilon>0$ be such that the ball $B\left(x_{0}, \varepsilon\right) \subset D$. We define

$$
\mathcal{L}_{x_{0}}^{\varepsilon}(u)=\frac{1}{\left|B\left(x_{0}, \varepsilon\right)\right|} \int_{B\left(x_{0}, \varepsilon\right)} u(x) \mathrm{d} x .
$$

The theorem claims that we can reach in finite time all the values for the spatial mean for the process $\left(Q u_{\varphi}(\cdot, t)\right)_{t \in \mathbb{R}^{+}}$.

Proof. With the hypotheses on $\mathcal{L}$, we have, for all $t \in \mathbb{R}^{+}$,

$$
v_{1}(t) \leqslant \mathcal{L}\left(u_{\varphi}(\cdot, t)\right) \leqslant v_{2}(t)
$$

$\mathbb{P}$-a.s. We fix $y \in\left(u_{0}, u_{1}\right)$. By the proof of Theorem 2.12, for almost all $\omega \in \Omega$, and for all $n \in \mathbb{N}^{*}$, there exist

$$
s_{n}(\omega) \text { such that } v_{2}\left(s_{n}(\omega), \omega\right)=u_{0}+\frac{1}{n}<y
$$

and

$$
t_{n}(\omega) \text { such that } v_{1}\left(t_{n}(\omega), \omega\right)=u_{1}-\frac{1}{n}>y
$$


Then

and

$$
\mathcal{L}\left(u_{\varphi}\left(\cdot, s_{n}(\omega), \omega\right)\right) \leqslant u_{0}+\frac{1}{n}
$$

$$
\mathcal{L}\left(u_{\varphi}\left(\cdot, t_{n}(\omega), \omega\right)\right) \geqslant u_{1}-\frac{1}{n}
$$

By continuity of the process $\left(\mathcal{L}\left(u_{\varphi}(\cdot, t)\right)\right)_{t \in \mathbb{R}^{+}}$, there exists $t \in\left[t_{n}(\omega) \wedge s_{n}(\omega), t_{n}(\omega) \vee s_{n}(\omega)\right]$ such that

$$
\mathcal{L}\left(u_{\varphi}(\cdot, t)\right)=y
$$

Theorem 2.15. In the case $(C)$, both steady states $u_{0}$ and $u_{1}$ are unstable in probability.

Remark 2.16. In this case, the steady states $u_{0}$ and $u_{1}$ repel the random field simultaneously away.

Proof. We prove that $u_{1}$ is unstable in probability. Since

$$
\sup _{t \in \mathbb{R}^{+}}\left|v_{2}(t)-u_{1}\right| \leqslant \sup _{t \in \mathbb{R}^{+}}\left\|u_{\varphi}(\cdot, t)-u_{1}\right\|_{\infty} \mathbb{P} \text {-a.s. }
$$

for any $\varepsilon>0$ we have

$$
\mathbb{P}\left\{\sup _{t \in \mathbb{R}^{+}}\left\|u_{\varphi}(\cdot, t)-u_{1}\right\|_{\infty}>\varepsilon\right\} \geqslant \mathbb{P}\left\{\sup _{t \in \mathbb{R}^{+}}\left|v_{2}(t)-u_{1}\right|>\varepsilon\right\}
$$

Let $b$ be a real such that $u_{1}-\varepsilon<\varphi_{2} \leqslant b<u_{1}$. Let $\tau_{u_{1}-\varepsilon, b}$ be the exit time from the interval $\left(u_{1}-\varepsilon, b\right)$ for the process $\left(v_{2}(t)\right)_{t \in \mathbb{R}^{+}}$

$$
\tau_{u_{1}-\varepsilon, b}=\inf \left\{t \geqslant 0: v_{2}(t) \notin\left(u_{1}-\varepsilon, b\right)\right\} .
$$

We use Ikeda and Watanabe [11], page 362, and Proposition 2.3 in order to obtain

$$
\begin{aligned}
\mathbb{P}\left\{\sup _{t \geqslant 0}\left|v_{2}(t)-u_{1}\right|>\varepsilon\right\} & \geqslant \mathbb{P}\left\{v_{2}\left(\tau_{u_{1}-\varepsilon, b}\right)=u_{1}-\varepsilon\right\} \\
& \geqslant \frac{F(b)-F\left(\varphi_{2}\right)}{F(b)-F\left(u_{1}-\varepsilon\right)} \underset{b \rightarrow u_{1}}{\longrightarrow} 1
\end{aligned}
$$

Hence $u_{1}$ is unstable in probability and analogous computations show that $u_{0}$ is also unstable in probability.

\subsection{Stability in probability in the case (D)}

Before studying the case (D) in details in the next section, the following theorem states the properties of stability in probability of the two steady states $u_{0}$ and $u_{1}$. The proof is similar to the proof of Theorem 2.10 , and it is again a consequence of monotonicity methods.

Theorem 2.17. In the case $(D)$, both steady states $u_{0}$ and $u_{1}$ are stable in probability.

\section{Purely Random attractor}

The main result of this section is that the random field $\left(u_{\varphi}(\cdot, t)\right)_{t \in \mathbb{R}^{+}}$converges to a Bernoulli random variable with values in $\left\{u_{0}, u_{1}\right\}$ (see Th. 3.5 below). This kind of behaviour appears only in the more difficult case (D) and this ends the monotonicity methods. Indeed, if we use the same technics as in the previous section, we can only have the following estimates:

$$
\mathbb{P}\left\{\lim _{t \rightarrow+\infty}\left\|u_{\varphi}(\cdot, t)-u_{0}\right\|_{\infty}=0\right\} \geqslant \frac{F\left(u_{1}\right)-F\left(\varphi_{2}\right)}{F\left(u_{1}\right)-F\left(u_{0}\right)}>0
$$


and

$$
\mathbb{P}\left\{\lim _{t \rightarrow+\infty}\left\|u_{\varphi}(\cdot, t)-u_{1}\right\|_{\infty}=0\right\} \geqslant \frac{F\left(\varphi_{1}\right)-F\left(u_{0}\right)}{F\left(u_{1}\right)-F\left(u_{0}\right)}>0
$$

Unfortunately the sum of the two above probabilities is equal to 1 if and only if $\varphi_{1}=\varphi_{2}$, which is out of interest. From now on, we give up the monotonicity methods and we will be able to solve our problem only when the ellipticity constant $k_{1}$ is large enough (see Hypothesis $(\mathrm{K})$ ). This restriction appears in the exponential decay estimate (see Prop. 3.1) between the random field and its spatial average.

We introduce the positive linear continuous operator $Q$ defined on $L^{2}(D)$ by

$$
Q f=\frac{1}{|D|} \int_{D} f(x) \mathrm{d} x
$$

We note $Q h$ for the vector $\left(Q h_{1}, \ldots, Q h_{r}\right)$.

It is worth noting that all the information about the limit of $\left(u_{\varphi}(\cdot, t)\right)_{t \in \mathbb{R}^{+}}$will be contained in the process $\left(Q u_{\varphi}(\cdot, t)\right)_{t \in \mathbb{R}^{+}}$, which satisfies

$$
Q u_{\varphi}(\cdot, t)=Q \varphi(\cdot)+\int_{0}^{t} Q g\left(u_{\varphi}(\cdot, s)\right) \mathrm{d} s+\sum_{j=1}^{r} \int_{0}^{t} Q h_{j}\left(u_{\varphi}(\cdot, s)\right) \mathrm{d} W_{s}^{j}
$$

The above relation is a $\operatorname{SDE}$ when we choose $\varphi=\varphi_{1}$ or $\varphi=\varphi_{2}$ as initial conditions. In that case, we obtain equations (2.1) and (2.2).

Proposition 3.1. Assume $(K),(G),(H)$ and $(I)$ hold. Then, if $k_{1} \geqslant C_{D}\left(\left|g^{\prime}\right|_{\infty}+2\left|h^{\prime}\right|_{\infty}^{2}\right)=k^{*}$, there exists a constant $\alpha>0$ such that for all $t \in \mathbb{R}^{+}$,

$$
\mathbb{E}\left\|u_{\varphi}(\cdot, t)-Q u_{\varphi}(\cdot, t)\right\|_{2}^{2} \leqslant \exp (-\alpha t)\|\varphi-Q \varphi\|_{2}^{2}
$$

Here, $C_{D}$ denotes the constant appearing in Poincaré-Wirtinger's inequality (see Brézis [4]) and it only depends on the geometry of the domain $D$.

Remark 3.2. This result means that the random field stabilizes almost surely for large times around a spatially homogeneous random process.

Remark 3.3. The above result implies that for all $\gamma \in(0,1]$, we have

$$
\mathbb{E} \int_{0}^{+\infty}\left\|u_{\varphi}(\cdot, s)-Q u_{\varphi}(\cdot, s)\right\|_{2}^{2 \gamma} \mathrm{d} s<+\infty
$$

The proof is quite similar of the one given in Chueshov and Vuillermot [7]. Nevertheless, we give it for the convenience for the reader.

Proof. We first prove that there exists $k^{*}$ such that for all $k_{1} \geqslant k^{*}$, there exists $\alpha \in \mathbb{R}^{+}$such that

$$
\frac{\mathrm{d}}{\mathrm{d} t}\left(\mathbb{E}\left\|u_{\varphi}(\cdot, t)-Q u_{\varphi}(\cdot, t)\right\|_{2}^{2}\right)+\alpha \mathbb{E}\left\|u_{\varphi}(\cdot, t)-Q u_{\varphi}(\cdot, t)\right\|_{2}^{2} \leqslant 0
$$


for all $t \in \mathbb{R}^{+}$. The process $\left(u_{\varphi}(\cdot, t)-Q u_{\varphi}(\cdot, t)\right)_{t \in \mathbb{R}^{+}}$satisfies

$$
\left\{\begin{array}{l}
\mathrm{d}\left(u_{\varphi}(x, t)-Q u_{\varphi}(\cdot, t)\right)=\operatorname{div}\left(k(x, t) \nabla\left(u_{\varphi}(x, t)-Q u_{\varphi}(\cdot, t)\right)\right) \mathrm{d} t \\
\quad+\left(g\left(u_{\varphi}(x, t)\right)-Q g\left(u_{\varphi}(\cdot, t)\right)\right) \mathrm{d} t+\sum_{j=1}^{r}\left(h_{j}\left(u_{\varphi}(x, t)\right)-Q h_{j}\left(u_{\varphi}(\cdot, t)\right)\right) \mathrm{d} W_{t}^{j},(x, t) \in D \times \mathbb{R}_{*}^{+}, \\
u_{\varphi}(x, 0)-Q u_{\varphi}(\cdot, 0)=\varphi(x)-Q \varphi \quad\left(u_{0}-u_{1}, u_{1}-u_{0}\right), \quad x \in D, \\
\frac{\partial\left(u_{\varphi}(x, t)-Q u_{\varphi}(\cdot, t)\right)}{\partial n(k)}=0, \quad(x, t) \in \partial D \times \mathbb{R}^{+} .
\end{array}\right.
$$

We apply Itô's formula. Taking expectation yields

$$
\begin{aligned}
\mathbb{E}\left\|u_{\varphi}(\cdot, t)-Q u_{\varphi}(\cdot, t)\right\|_{2}^{2}= & \|\varphi-Q \varphi\|_{2}^{2}-2 \mathbb{E} \int_{0}^{t} \int_{D}\left\langle\nabla u_{\varphi}(x, s), k(x, s) \nabla u_{\varphi}(x, s)\right\rangle_{\mathbb{R}^{d}} \mathrm{~d} x \mathrm{~d} s \\
& +2 \mathbb{E} \int_{0}^{t} \int_{D}\left(u_{\varphi}(x, s)-Q u_{\varphi}(\cdot, s)\right)\left(g\left(u_{\varphi}(x, s)\right)-Q g\left(u_{\varphi}(\cdot, s)\right)\right) \mathrm{d} x \mathrm{~d} s \\
& +\mathbb{E} \int_{0}^{t} \int_{D}\left|h\left(u_{\varphi}(x, s)\right)-Q h\left(u_{\varphi}(\cdot, s)\right)\right|^{2} \mathrm{~d} x \mathrm{~d} s .
\end{aligned}
$$

Thanks to Poincaré-Wirtinger's inequality (see Brézis [4]), there exists a universal constant $C_{D}>0$ such that

$$
\mathbb{E}\left\|\nabla u_{\varphi}(\cdot, t)\right\|_{2}^{2} \geqslant \frac{1}{C_{D}} \mathbb{E}\left\|u_{\varphi}(\cdot, t)-Q u_{\varphi}(\cdot, t)\right\|_{2}^{2} .
$$

Using the ellipticity assumption (see Hypothesis (K)) and (3.8), (3.7) becomes

$$
\begin{aligned}
\mathbb{E}\left\|u_{\varphi}(\cdot, t)-Q u_{\varphi}(\cdot, t)\right\|_{2}^{2} \leqslant & \|\varphi-Q \varphi\|_{2}^{2}-2 \mathbb{E} \int_{0}^{t} k_{1} \frac{1}{C_{D}}\left\|u_{\varphi}(\cdot, s)-Q u_{\varphi}(\cdot, s)\right\|_{2}^{2} \mathrm{~d} s \\
& +2 \mathbb{E} \int_{0}^{t} \int_{D}\left(u_{\varphi}(x, s)-Q u_{\varphi}(\cdot, s)\right)\left(g\left(u_{\varphi}(x, s)\right)-Q g\left(u_{\varphi}(\cdot, s)\right)\right) \mathrm{d} x \mathrm{~d} s \\
& +\mathbb{E} \int_{0}^{t} \int_{D}\left|h\left(u_{\varphi}(x, s)\right)-Q h\left(u_{\varphi}(\cdot, s)\right)\right|^{2} \mathrm{~d} x \mathrm{~d} s .
\end{aligned}
$$

We estimate the second term of the right-hand side of relation (3.9). We have

$$
\begin{aligned}
& 2 \mathbb{E} \int_{D}\left(u_{\varphi}(x, t)-Q u_{\varphi}(\cdot, t)\right)\left(g\left(u_{\varphi}(x, t)\right)-Q g\left(u_{\varphi}(\cdot, t)\right)\right) \mathrm{d} x= \\
& 2 \mathbb{E} \int_{D}\left(u_{\varphi}(x, t)-Q u_{\varphi}(\cdot, t)\right)\left(g\left(u_{\varphi}(x, t)\right)-g\left(Q u_{\varphi}(\cdot, t)\right)\right) \mathrm{d} x \\
& \quad+\mathbb{E} \int_{D}\left(u_{\varphi}(x, t)-Q u_{\varphi}(\cdot, t)\right)\left(g\left(Q u_{\varphi}(\cdot, t)\right)-Q g\left(u_{\varphi}(\cdot, t)\right)\right) \mathrm{d} x,
\end{aligned}
$$

for all $t \in \mathbb{R}^{+}$. Note that $g\left(Q u_{\varphi}(\cdot, t)\right)$ and $Q g\left(u_{\varphi}(\cdot, t)\right)$ do not depend on $x$. So the second term of the right-hand side vanishes thanks to the definition of $Q$. Finally we obtain

$$
2\left|\mathbb{E} \int_{D}\left(u_{\varphi}(x, t)-Q u_{\varphi}(\cdot, t)\right)\left(g\left(u_{\varphi}(x, t)\right)-Q g\left(u_{\varphi}(\cdot, t)\right)\right) \mathrm{d} x\right| \leqslant 2\left|g^{\prime}\right|_{\infty} \mathbb{E}\left\|u_{\varphi}(\cdot, t)-Q u_{\varphi}(\cdot, t)\right\|_{2}^{2}
$$


Now we estimate the last term of the right-hand side of the relation (3.9), we have,

$$
\begin{aligned}
\mathbb{E} \int_{D}\left|h\left(u_{\varphi}(x, t)\right)-Q h\left(u_{\varphi}(\cdot, t)\right)\right|^{2} \mathrm{~d} x \leqslant & 2 \mathbb{E} \int_{D}\left|h\left(u_{\varphi}(x, t)\right)-h\left(Q u_{\varphi}(\cdot, t)\right)\right|^{2} \mathrm{~d} x \\
& +2 \mathbb{E} \int_{D}\left|h\left(Q u_{\varphi}(\cdot, t)\right)-Q h\left(u_{\varphi}(\cdot, t)\right)\right|^{2} \mathrm{~d} x \\
\leqslant & 2\left|h^{\prime}\right|_{\infty}^{2} \mathbb{E}\left\|u_{\varphi}(\cdot, t)-Q u_{\varphi}(\cdot, t)\right\|_{2}^{2}+2 \mathbb{E} \int_{D}\left|h\left(Q u_{\varphi}(\cdot, t)\right)-Q h\left(u_{\varphi}(\cdot, t)\right)\right|^{2} \mathrm{~d} x
\end{aligned}
$$

We write

$$
\begin{aligned}
\mathbb{E} \int_{D}\left|h\left(Q u_{\varphi}(\cdot, t)\right)-Q h\left(u_{\varphi}(\cdot, t)\right)\right|^{2} \mathrm{~d} x & =|D| \mathbb{E}\left|\frac{1}{|D|} \int_{D} h\left(Q u_{\varphi}(\cdot, t)\right)-h\left(u_{\varphi}(x, t)\right) \mathrm{d} x\right|^{2} \\
& \leqslant\left|h^{\prime}\right|_{\infty}^{2} \mathbb{E}\left\|u_{\varphi}(\cdot, t)-Q u_{\varphi}(\cdot, t)\right\|_{2}^{2}
\end{aligned}
$$

and we replace it into (3.11) to obtain

$$
\mathbb{E} \int_{D}\left|h\left(u_{\varphi}(x, t)\right)-Q h\left(u_{\varphi}(\cdot, t)\right)\right|^{2} \mathrm{~d} x \leqslant 4\left|h^{\prime}\right|_{\infty}^{2} \mathbb{E}\left\|u_{\varphi}(\cdot, t)-Q u_{\varphi}(\cdot, t)\right\|_{2}^{2}
$$

Finally, injecting relations (3.10) and (3.12) in the relation (3.9), we get

$$
\begin{aligned}
\frac{\mathrm{d}}{\mathrm{d} t} \mathbb{E}\left\|u_{\varphi}(\cdot, t)-Q u_{\varphi}(\cdot, t)\right\|_{2}^{2} & \leqslant\left(-\frac{2 k_{1}}{C_{D}}+2\left|g^{\prime}\right|_{\infty}+4\left|h^{\prime}\right|_{\infty}^{2}\right) \mathbb{E}\left\|u_{\varphi}(\cdot, t)-Q u_{\varphi}(\cdot, t)\right\|_{2}^{2} \\
& \leqslant-\alpha \mathbb{E}\left\|u_{\varphi}(\cdot, t)-Q u_{\varphi}(\cdot, t)\right\|_{2}^{2}
\end{aligned}
$$

if we choose $\alpha>0$ so that $-\frac{2 k_{1}}{C_{D}}+2\left|g^{\prime}\right|_{\infty}+4\left|h^{\prime}\right|_{\infty}^{2}<-\alpha<0$ with $k_{1} \geqslant k^{*}:=C_{D}\left(\left|g^{\prime}\right|_{\infty}+2\left|h^{\prime}\right|_{\infty}^{2}\right)$, relation $(3.13)$ is exactly (3.6). Integrating relation (3.13) between 0 and $t$ gives the result, namely (3.4).

The asymptotic behaviour of $\left(u_{\varphi}(\cdot, t)\right)_{t \in \mathbb{R}^{+}}$is then quite close to the asymptotic behaviour of the random process $\left(Q u_{\varphi}(\cdot, t)\right)_{t \in \mathbb{R}^{+}}$. We specify its long-time behaviour in the following theorem.

Theorem 3.4. Assume Hypotheses $(K),(G),(H)$ and $(I)$ hold. In addition, we assume that $k_{1} \geqslant k^{*}$ (see Prop. 3.1). Then, in the case (D), there exists a real number $Z$ such that

$$
\mathbb{P}\left\{\lim _{t \rightarrow+\infty} Q u_{\varphi}(\cdot, t)=u_{0}\right\}=\frac{F\left(u_{1}\right)-F(Q \varphi)}{F\left(u_{1}\right)-F\left(u_{0}\right)}-\frac{Z}{F\left(u_{1}\right)-F\left(u_{0}\right)}
$$

and

$$
\mathbb{P}\left\{\lim _{t \rightarrow+\infty} Q u_{\varphi}(\cdot, t)=u_{1}\right\}=\frac{F(Q \varphi)-F\left(u_{0}\right)}{F\left(u_{1}\right)-F\left(u_{0}\right)}+\frac{Z}{F\left(u_{1}\right)-F\left(u_{0}\right)}
$$

where $F$ is a Feller function (see Relation (2.8)).

The technics used in the proof of this result are similar to those used in the classical theory of SDE's. The proof is quite long and postponed in the following section.

We now give the main result of this section. This theorem shows that the random field converges to a random variable taking its values in $\left\{u_{0}, u_{1}\right\}$.

Theorem 3.5. Assume that $(K),(G),(H)$ and $(I)$ hold. In addition, we assume that the ellipticity constant $k_{1} \geqslant k^{*}$ (see Prop. 3.1) and that the coefficients satisfy the relation of the case (D) (see Prop. 2.3). Let $\xi$ be the random variable defined by

$$
\xi=u_{0} \mathbb{1}_{\Omega_{0}}+u_{1} \mathbb{1}_{\Omega_{1}}
$$




$$
\begin{gathered}
\text { where } \Omega_{0}=\left\{\lim _{t \rightarrow+\infty} Q u_{\varphi}(\cdot, t)=u_{0}\right\} \text { and } \Omega_{1}=\left\{\lim _{t \rightarrow+\infty} Q u_{\varphi}(\cdot, t)=u_{1}\right\} . \text { Then we have } \\
\mathbb{E}\left\|u_{\varphi}(\cdot, t)-\xi\right\|_{2}^{2} \underset{t \rightarrow+\infty}{\longrightarrow} 0 .
\end{gathered}
$$

More precisely, there exists a real number $Z$ (the constant appearing in Th. 3.4) and a sequence $\left(t_{n}\right)_{n \in \mathbb{N}}$ which tends to $+\infty$ such that

$$
\mathbb{P}\left\{\lim _{n \rightarrow+\infty}\left\|u_{\varphi}\left(\cdot, t_{n}\right)-u_{0}\right\|_{2}=0\right\}=\frac{F\left(u_{1}\right)-F(Q \varphi)}{F\left(u_{1}\right)-F\left(u_{0}\right)}-\frac{Z}{F\left(u_{1}\right)-F\left(u_{0}\right)}
$$

and

$$
\mathbb{P}\left\{\lim _{n \rightarrow+\infty}\left\|u_{\varphi}\left(\cdot, t_{n}\right)-u_{1}\right\|_{2}=0\right\}=\frac{F(Q \varphi)-F\left(u_{0}\right)}{F\left(u_{1}\right)-F\left(u_{0}\right)}+\frac{Z}{F\left(u_{1}\right)-F\left(u_{0}\right)} .
$$

Remark 3.6. This theorem is a improvement of Theorem 2.7 of Chueshov and Vuillermot [7]. Indeed the authors investigate only the case when the sign of the drift coefficient $g$ is constant. This result specify one of the alternative behaviour described in Hetzer et al. [9]. Unfortunately, we are not able to get rid of the assumption on the ellipticity constant.

Proof. Using the relation (3.1) and the definition of $\xi$, we have

$$
\begin{aligned}
\mathbb{E}\left\|u_{\varphi}(\cdot, t)-\xi\right\|_{2}^{2} \leqslant & 2 \mathbb{E}\left\|u_{\varphi}(\cdot, t)-Q u_{\varphi}(\cdot, t)\right\|_{2}^{2}+2 \mathbb{E}\left\|Q u_{\varphi}(\cdot, t)-\xi\right\|_{2}^{2} \\
\leqslant & 2 \mathrm{e}^{-\alpha t}\|\varphi-Q \varphi\|_{2}^{2} \\
& +2|D| \mathbb{E}\left|\left(Q u_{\varphi}(\cdot, t)-u_{0}\right) \mathbb{1}_{\Omega_{0}}\right|^{2}+2|D| \mathbb{E}\left|\left(Q u_{\varphi}(\cdot, t)-u_{1}\right) \mathbb{1}_{\Omega_{1}}\right|^{2} .
\end{aligned}
$$

Thanks to Theorem 3.4 and the dominated convergence theorem, we infer that (3.16) holds.

Now we write

$$
\left\|u_{\varphi}(\cdot, t)-u_{0}\right\|_{2}^{2} \leqslant 2\left\|u_{\varphi}(\cdot, t)-Q u_{\varphi}(\cdot, t)\right\|_{2}^{2}+2\left\|Q u_{\varphi}(\cdot, t)-u_{0}\right\|_{2}^{2} .
$$

We deduce there exists a sequence $\left(t_{n}\right)_{n \in \mathbb{N}} \underset{n \rightarrow+\infty}{\longrightarrow}+\infty$ such that

$$
\lim _{n \rightarrow+\infty}\left\|u_{\varphi}\left(\cdot, t_{n}\right)-u_{0}\right\|_{2}^{2} \leqslant \lim _{n \rightarrow+\infty} 2\left\|u_{\varphi}\left(\cdot, t_{n}\right)-Q u_{\varphi}\left(\cdot, t_{n}\right)\right\|_{2}^{2}+2 \lim _{n \rightarrow+\infty}\left\|Q u_{\varphi}\left(\cdot, t_{n}\right)-u_{0}\right\|_{2}^{2} .
$$

Using (3.14), we get

$$
\mathbb{P}\left\{\lim _{n \rightarrow+\infty}\left\|u_{\varphi}\left(\cdot, t_{n}\right)-u_{0}\right\|_{2}^{2}=0\right\} \geqslant \frac{F\left(u_{1}\right)-F(Q \varphi)}{F\left(u_{1}\right)-F\left(u_{0}\right)}-\frac{Z}{F\left(u_{1}\right)-F\left(u_{0}\right)} .
$$

In a similar way, we can show that

$$
\mathbb{P}\left\{\lim _{n \rightarrow+\infty}\left\|u_{\varphi}\left(\cdot, t_{n}\right)-u_{0}\right\|_{2}^{2}=0\right\} \geqslant \frac{F(Q \varphi)-F\left(u_{0}\right)}{F\left(u_{1}\right)-F\left(u_{0}\right)}+\frac{Z}{F\left(u_{1}\right)-F\left(u_{0}\right)} .
$$

Since the sum of these two probabilities is 1 , it is clear that we have (3.17) and (3.18).

\section{Asymptotic Behaviour of the spatial aVerage}

In this section we prove Theorem 3.4 stated in the previous one. We need several auxiliary results. We begin with a simple remark about the functions $g$ and $h_{j}$ 's. 
Remark 4.1. Let $G$ the function defined for all $u \in\left[u_{0}, u_{1}\right]$ by $G(u)=c\left(u-u_{0}\right)\left(u_{1}-u\right)$ where $c$ is a constant such that $|g(u)|+\sum_{j=1}^{r}\left|h_{j}(u)\right| \leqslant G(u)$. Using the concavity of $G$ and Jensen's inequality, we have, for all $s \in \mathbb{R}^{+}$,

$$
\begin{aligned}
\left|Q g\left(u_{\varphi}(\cdot, s)\right)-g\left(Q u_{\varphi}(\cdot, s)\right)\right| & \leqslant\left|Q g\left(u_{\varphi}(\cdot, s)\right)\right|+\left|G\left(Q u_{\varphi}(\cdot, s)\right)\right| \\
& \leqslant\left|Q G\left(u_{\varphi}(\cdot, s)\right)\right|+G\left(Q u_{\varphi}(\cdot, s)\right) \\
& \leqslant 2 G\left(Q u_{\varphi}(\cdot, s)\right) .
\end{aligned}
$$

The same holds with the functions $h_{j}$ 's. It is clear that the left-hand side of (4.1) is bounded. This estimation clarify the local behaviour of $Q g\left(u_{\varphi}(\cdot, s)\right)-g\left(Q u_{\varphi}(\cdot, s)\right)$ when $Q u_{\varphi}(\cdot, s)$ is close to $u_{0}$ or $u_{1}$.

Lemma 4.2. Assume hypothesis $(G)$ (resp. $(H))$. Then there exists a constant $C$ such that for all $t>0$,

$$
\left|Q g\left(u_{\varphi}(\cdot, t)\right)-g\left(Q u_{\varphi}(\cdot, t)\right)\right| \leqslant C\left\|u_{\varphi}(\cdot, t)-Q u_{\varphi}(\cdot, t)\right\|_{2}^{2}
$$

almost surely (resp. $h_{j}$ instead of $g$ ).

Proof. We only prove the result for the function $g$. Using a Taylor expansion around the point $Q u_{\varphi}(\cdot, t)$ there exists a measurable function $\lambda \in(0,1)$ depending on $x, t$ and $\omega$ such that for all $t>0$

$$
\begin{aligned}
Q g\left(u_{\varphi}(\cdot, t)\right)-g\left(Q u_{\varphi}(\cdot, t)\right)= & \frac{1}{|D|} \int_{D} g\left(u_{\varphi}(x, t)\right)-g\left(Q u_{\varphi}(\cdot, t)\right) \mathrm{d} x \\
= & \frac{1}{|D|} \int_{D} g^{\prime}\left(Q u_{\varphi}(\cdot, t)\right)\left(u_{\varphi}(x, t)-Q u_{\varphi}(\cdot, t)\right) \mathrm{d} x \\
& +\frac{1}{|D|} \int_{D} g^{\prime \prime}\left(\lambda u_{\varphi}(x, t)+(1-\lambda) Q u_{\varphi}(\cdot, t)\right)\left(u_{\varphi}(x, t)-Q u_{\varphi}(\cdot, t)\right)^{2} \mathrm{~d} x
\end{aligned}
$$

almost surely. Since the first term of the right-hand side is zero by the definition of the operator $Q$, we have the result with $C=\frac{\left|g^{\prime \prime}\right|_{\infty}}{|D|}$.

For $u_{0}<a<b<u_{1}$ and $\varphi$ satisfying hypothesis (I) and $a<Q \varphi<b$, let us define

$$
\tau_{a, b}=\inf \left\{t>0: Q u_{\varphi}(\cdot, t) \notin[a, b]\right\}
$$

the first exit time from the interval $[a, b]$. Arguing as in Ikeda and Watanabe [11], we first compute the probability that the process $\left(Q u_{\varphi}(\cdot, t)\right)_{t \in \mathbb{R}^{+}}$leaves the interval $(a, b)$ by the left side (namely the point $a$ ) and the probability that the process goes out from this interval by the point $b$. In order to do this, we need that $\tau_{a, b}$ is almost surely finite which is the purpose of the Proposition 4.5 below.

Proposition 4.3. Assume hypotheses (K), $(G),(H)$ and (I) hold. In addition we assume that $k_{1} \geqslant k^{*}$ (see Prop. 3.1) and that the coefficients satisfy the relation of the case (D). Then there exists a random variable $R(a, b) \in L^{1}(\Omega)$ such that

$$
\mathbb{P}\left\{Q u_{\varphi}\left(\cdot, \tau_{a, b}\right)=a\right\}=\frac{F(b)-F(Q \varphi)}{F(b)-F(a)}-\frac{\mathbb{E} R(a, b)}{F(b)-F(a)}
$$

and

$$
\mathbb{P}\left\{Q u_{\varphi}\left(\cdot, \tau_{a, b}\right)=b\right\}=\frac{F(Q \varphi)-F(a)}{F(b)-F(a)}+\frac{\mathbb{E} R(a, b)}{F(b)-F(a)}
$$


Proof. The proof will be divided into several steps. We first introduce an auxiliary random process. We apply Itô's formula to a Feller function $F$ and we obtain for all $t>0$

$$
\begin{aligned}
F\left(Q u_{\varphi}(\cdot, t)\right)= & F(\varphi)+\int_{0}^{t \wedge \tau_{a, b}} F^{\prime}\left(Q u_{\varphi}(\cdot, s)\right) Q h\left(u_{\varphi}(\cdot, s)\right) \mathrm{d} W_{s} \\
& +\int_{0}^{t \wedge \tau_{a, b}} F^{\prime}\left(Q u_{\varphi}(\cdot, s)\right) Q g\left(u_{\varphi}(\cdot, s)\right)+\frac{F^{\prime \prime}\left(Q u_{\varphi}(\cdot, s)\right)}{2}\left|Q h\left(u_{\varphi}(\cdot, s)\right)\right|^{2} \mathrm{~d} s
\end{aligned}
$$

So the random process $(R(t ; a, b))_{t \in \mathbb{R}^{+}}$defined by

$$
R(t ; a, b)=\int_{0}^{t \wedge \tau_{a, b}} \frac{1}{2} F^{\prime \prime}\left(Q u_{\varphi}(\cdot, s)\right)\left|Q h\left(u_{\varphi}(\cdot, s)\right)\right|^{2}+F^{\prime}\left(Q u_{\varphi}(\cdot, s)\right) Q g\left(u_{\varphi}(\cdot, s)\right) \mathrm{d} s
$$

arises naturally. We now focus on it.

Step 1. For all $t_{1}, t_{2} \in \mathbb{R}^{+}$, we define $\Delta\left(t_{1}, t_{2} ; a, b\right)=R\left(t_{2} ; a, b\right)-R\left(t_{1} ; a, b\right)$ and we show that there exist $c>0$ and $\gamma \in(0,1]$ such that almost surely

$$
\left|\Delta\left(t_{1}, t_{2} ; a, b\right)\right| \leqslant c \int_{t_{1} \wedge \tau_{a, b}}^{t_{2} \wedge \tau_{a, b}}\left\|u_{\varphi}(\cdot, s)-Q u_{\varphi}(\cdot, s)\right\|_{2}^{2 \gamma} \mathrm{d} s
$$

Indeed, replacing $F^{\prime \prime}$ from relation (2.7) into (4.5), we have for all $t_{1}, t_{2} \in \mathbb{R}^{+}$,

$$
\begin{aligned}
\Delta\left(t_{1}, t_{2} ; a, b\right) & =\int_{t_{1} \wedge \tau_{a, b}}^{t_{2} \wedge \tau_{a, b}}-\frac{g\left(Q u_{\varphi}(\cdot, s)\right)}{\left|h\left(Q u_{\varphi}(\cdot, s)\right)\right|^{2}} F^{\prime}\left(Q u_{\varphi}(\cdot, s)\right)\left|Q h\left(u_{\varphi}(\cdot, s)\right)\right|^{2}+F^{\prime}\left(Q u_{\varphi}(\cdot, s)\right) Q g\left(u_{\varphi}(\cdot, s)\right) \mathrm{d} s \\
& =\int_{t_{1} \wedge \tau_{a, b}}^{t_{2} \wedge \tau_{a, b}} \Delta^{1}(s)+\Delta^{2}(s) \mathrm{d} s
\end{aligned}
$$

P-a.s. with

$$
\Delta^{1}(s)=F^{\prime}\left(Q u_{\varphi}(\cdot, s)\right)\left(Q g\left(u_{\varphi}(\cdot, s)\right)-g\left(Q u_{\varphi}(\cdot, s)\right)\right)
$$

and

$$
\begin{aligned}
\Delta^{2}(s) & =F^{\prime}\left(Q u_{\varphi}(\cdot, s)\right)\left(g\left(Q u_{\varphi}(\cdot, s)\right)-\frac{g\left(Q u_{\varphi}(\cdot, s)\right)}{\left|h\left(Q u_{\varphi}(\cdot, s)\right)\right|^{2}}\left|Q h\left(u_{\varphi}(\cdot, s)\right)\right|^{2}\right) \\
& =\frac{F^{\prime}\left(Q u_{\varphi}(\cdot, s)\right) g\left(Q u_{\varphi}(\cdot, s)\right)}{\left|h\left(Q u_{\varphi}(\cdot, s)\right)\right|^{2}}\left(\left|h\left(Q u_{\varphi}(\cdot, s)\right)\right|^{2}-\left|Q h\left(u_{\varphi}(\cdot, s)\right)\right|^{2}\right) .
\end{aligned}
$$

We first estimate $\Delta^{1}$. We have for $\gamma \in(0,1)$ to be fixed later,

$$
\begin{aligned}
\left|\Delta^{1}(s)\right| & =\left|F^{\prime}\left(Q u_{\varphi}(\cdot, s)\right) \| Q g\left(u_{\varphi}(\cdot, s)\right)-g\left(Q u_{\varphi}(\cdot, s)\right)\right|^{1-\gamma}\left|Q g\left(u_{\varphi}(\cdot, s)\right)-g\left(Q u_{\varphi}(\cdot, s)\right)\right|^{\gamma} \\
& \left.\leqslant 2 c\left|F^{\prime}\left(Q u_{\varphi}(\cdot, s)\right)\right| G\left(Q u_{\varphi}(\cdot, s)\right)^{1-\gamma} \| u_{\varphi}(\cdot, s)-Q u_{\varphi}(\cdot, s)\right) \|_{2}^{2 \gamma}
\end{aligned}
$$

where we have used (4.1) and (4.2). We show that the continuous function $u \mapsto 2 F^{\prime}(u) G(u)^{1-\gamma}$ is bounded. The problem lies around $u_{0}$ and $u_{1}$. But in a neighborhood of $u_{0}$ we have the following equivalence

$$
2 F^{\prime}(u) G(u) \sim_{u_{0}} c\left(u-u_{0}\right)^{-2 \frac{g^{\prime}\left(u_{0}\right)}{\left|h^{\prime}\left(u_{0}\right)\right|^{2}}}\left(u-u_{0}\right)^{1-\gamma}
$$


So, if $g^{\prime}\left(u_{0}\right)<0$ then $-\frac{2 g^{\prime}\left(u_{0}\right)}{\left|h^{\prime}\left(u_{0}\right)\right|^{2}}>0$ and the function is bounded (we fix $\gamma=1$ ). But if not, keeping in mind that we are in the case $(\mathrm{D})$, we may choose $0<\gamma<1-\frac{2 g^{\prime}\left(u_{0}\right)}{\left|h^{\prime}\left(u_{0}\right)\right|^{2}}$ and the function is bounded around $u_{0}$. Similar arguments are valid around $u_{1}$. So we choose $\gamma$ such that

$$
\Delta^{1}(s) \leqslant c\left\|u_{\varphi}(\cdot, s)-Q u_{\varphi}(\cdot, s)\right\|_{2}^{2 \gamma}
$$

$\mathbb{P}$-a.s. Now we look at $\Delta^{2}$ closer. Using (4.1) and (4.2), we estimate $\left|\Delta^{2}(s)\right|$,

$$
\begin{aligned}
\left|\Delta^{2}(s)\right|= & \sum_{j=1}^{r} \frac{\left|F^{\prime}\left(Q u_{\varphi}(\cdot, s)\right)\right|\left|g\left(Q u_{\varphi}(\cdot, s)\right)\right|}{\left|h\left(Q u_{\varphi}(\cdot, s)\right)\right|^{2}}\left|h_{j}\left(Q u_{\varphi}(\cdot, s)\right)+Q h_{j}\left(u_{\varphi}(\cdot, s)\right)\right| \\
& \times\left|h_{j}\left(Q u_{\varphi}(\cdot, s)\right)-Q h_{j}\left(u_{\varphi}(\cdot, s)\right)\right| \\
\leqslant & \sum_{j=1}^{r} 2 c \frac{|g(Q u(\cdot, s))|}{\left|h\left(Q u_{\varphi}(\cdot, s)\right)\right|^{2}} G\left(Q u_{\varphi}(\cdot, s)\right)\left|F^{\prime}\left(Q u_{\varphi}(\cdot, s)\right)\right| \\
& \times\left|h_{j}\left(Q u_{\varphi}(\cdot, s)\right)-Q h_{j}\left(u_{\varphi}(\cdot, s)\right)\right|^{1-\gamma}\left\|u_{\varphi}(\cdot, s)-Q u_{\varphi}(\cdot, s)\right\|_{2}^{2 \gamma} .
\end{aligned}
$$

Around $u_{0}$, the function $u \mapsto \frac{|g(u)|}{|h(u)|^{2}} 2 G(u)$ is equivalent to

$$
2 \frac{\left(u-u_{0}\right)\left|g^{\prime}\left(u_{0}\right)\right|}{\left|h^{\prime}\left(u_{0}\right)\right|^{2}\left(u-u_{0}\right)^{2}} G^{\prime}\left(u_{0}\right)\left(u-u_{0}\right)
$$

so it is bounded. Similar arguments are valid around $u_{1}$. If we choose the same $\gamma$ as in (4.10) we obtain that

$$
\left|\Delta^{2}(s)\right| \leqslant c\left\|u_{\varphi}(\cdot, s)-Q u_{\varphi}(\cdot, s)\right\|_{2}^{2 \gamma}
$$

$\mathbb{P}$-a.s. for all $s \in \mathbb{R}^{+}$. Using (4.10) and (4.11), we obtain (4.6) and the step 1 is proved.

Step 2. There exists a random variable $R(a, b) \in L^{1}(\Omega)$ such that

$$
R(t ; a, b) \underset{t \rightarrow+\infty}{\longrightarrow} R(a, b) \text { in } L^{1}(\Omega) .
$$

Indeed, using the dominated convergence theorem and (3.5) we get

$$
\begin{gathered}
\mathbb{E}\left|\Delta\left(t_{1}, t_{2} ; a, b\right)\right| \underset{t_{1}, \overrightarrow{t_{2} \rightarrow+\infty}}{\longrightarrow} \quad c \mathbb{E} \int_{t_{1} \wedge \tau_{a, b}}^{t_{2} \wedge \tau_{a, b}}\left\|u_{\varphi}(\cdot, s)-Q u_{\varphi}(\cdot, s)\right\|_{2}^{2 \gamma} \mathrm{d} s \\
\end{gathered}
$$

Then it is sufficient to take $R(a, b)$ as the strong limit of $R(t ; a, b)$ in $L^{1}(\Omega)$.

Step 3. We prove (4.3) and (4.4). We admit for the moment that $\tau_{a, b}$ is finite almost surely (this will be proved in Prop. 4.5 below). Since $F$ is a bounded continuous function (in the case (D)), the following relation holds almost surely

$$
F\left(Q u_{\varphi}\left(\cdot, \tau_{a, b} \wedge t\right)\right) \underset{t \rightarrow+\infty}{\longrightarrow} F\left(Q u_{\varphi}\left(\cdot, \tau_{a, b}\right)\right)
$$

The dominated convergence theorem yields

$$
\mathbb{E} F\left(Q u_{\varphi}\left(\cdot, \tau_{a, b} \wedge t\right)\right) \underset{t \rightarrow+\infty}{\longrightarrow} \mathbb{E} F\left(Q u_{\varphi}\left(\cdot, \tau_{a, b}\right)\right)=F(a) \mathbb{P}\left\{F\left(Q u_{\varphi}\left(\tau_{a, b}\right)\right)=F(a)\right\}+F(b) \mathbb{P}\left\{F\left(Q u_{\varphi}\left(\tau_{a, b}\right)\right)=F(b)\right\}
$$


Taking expectation in (4.5) and letting $t$ tend to $+\infty$, we obtain

$$
\mathbb{E} F\left(Q u_{\varphi}\left(\cdot, \tau_{a, b}\right)\right)=F(Q \varphi)+\mathbb{E} R(a, b) .
$$

Since $F$ is one-to-one, we infer that

$$
F(a) \mathbb{P}\left\{Q u_{\varphi}\left(\tau_{a, b}\right)=a\right\}+F(b) \mathbb{P}\left\{Q u_{\varphi}\left(\tau_{a, b}\right)=b\right\}=F(Q \varphi)+\mathbb{E} R(a, b) .
$$

Since $\mathbb{P}\left\{\tau_{a, b}<+\infty\right\}=1$ (see Prop. 4.5), it holds that $\mathbb{P}\left\{Q u_{\varphi}\left(\cdot, \tau_{a, b}\right)=a\right\}+\mathbb{P}\left\{Q u_{\varphi}\left(\cdot, \tau_{a, b}\right)=b\right\}=1$ and the result is proved.

Remark 4.4. If the sign of the coefficient $g$ is constant, then the limit of $R(t ; a, b)$ as $t \rightarrow \infty$ can be easily deduced from monotone arguments. This is one of the reason why this proposition seemed to be less important in Chueshov and Vuillermot [7] and was totally useless in Chueshov and Vuillermot [6] and Bergé et al. [3]. Actually, in Chueshov and Vuillermot [6], Chueshov and Vuillermot [7] and Bergé et al. [3], the asymptotic behaviour of $\left(Q u_{\varphi}(\cdot, t)\right)_{t \in \mathbb{R}^{+}}$follows trivially from a martingale convergence theorem.

It remains to prove the following proposition.

Proposition 4.5. Assume hypotheses $(G),(H),(K)$ and (I) hold. We assume again that $k_{1} \geqslant k^{*}$ (see Prop. 3.1) and that the coefficients satisfy the relation of the case $(D)$. Then we have

$$
\mathbb{E} \tau_{a, b}<+\infty
$$

in particular $\mathbb{P}\left\{\tau_{a, b}<+\infty\right\}=1$.

Remark 4.6. In Chueshov and Vuillermot [7], the exit time $\tau_{a, b}$ is trivially almost surely finite since the authors first prove that the limit of the process $\left(Q u_{\varphi}(\cdot, t)\right)_{t \in \mathbb{R}^{+}}$takes its values in $\left\{u_{0}, u_{1}\right\}$. Thus the process leaves each intervals $(a, b)$ in finite time. However, they need the finiteness of this exit time in a similar way.

Proof. We first introduce the function $H$, solution of the following differential equation

$$
\frac{1}{2}|h(u)|^{2} H^{\prime \prime}(u)+g(u) H^{\prime}(u)=|h(u)| .
$$

Simple computations allow us to write the function $H$ explicitly

$$
H(u)=-\int^{u} \frac{F(s) w(s)}{F^{\prime}(s)} \mathrm{d} s+F(u) \int^{u} \frac{w(s)}{F^{\prime}(s)} \mathrm{d} s+c_{1}+c_{2} F(u)
$$

where $w(s)=\frac{2}{|h(s)|}$ and $F$ is the Feller function (Relation (2.7)).

We have

$$
H^{\prime}(u)=F^{\prime}(u) \int^{u} \frac{w(s)}{F^{\prime}(s)} \mathrm{d} s
$$

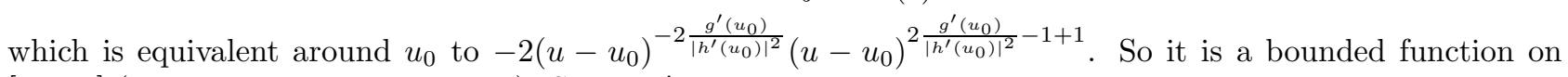
$\left[u_{0}, u_{1}\right]$ (the study near $u_{1}$ is similar). Since $H^{\prime}$ is bounded, $H$ is also bounded.

We turn to the proof of the relation (4.12). We apply Itô's formula to $\left(Q u_{\varphi}(\cdot, t)\right)_{t \in \mathbb{R}^{+}}$with the function $H$. This yields

$$
\begin{aligned}
H\left(Q u_{\varphi}\left(\cdot, t \wedge \tau_{a, b}\right)\right)= & H(Q \varphi)+\int_{0}^{t \wedge \tau_{a, b}} \frac{1}{2} H^{\prime \prime}\left(Q u_{\varphi}(\cdot, s)\right)\left|Q h\left(u_{\varphi}(\cdot, s)\right)\right|^{2}+H^{\prime}\left(Q u_{\varphi}(\cdot, s)\right) Q g\left(u_{\varphi}(\cdot, s)\right) \mathrm{d} s \\
& +\int_{0}^{t \wedge \tau_{a, b}} H^{\prime}\left(Q u_{\varphi}(\cdot, s)\right) Q h\left(u_{\varphi}(\cdot, s)\right) \mathrm{d} W_{s} .
\end{aligned}
$$


We use the relation (4.13) and we obtain

$$
\begin{aligned}
\mathbb{E} H\left(Q u_{\varphi}\left(\cdot, t \wedge \tau_{a, b}\right)\right)= & H(Q \varphi)+\mathbb{E} \int_{0}^{t \wedge \tau_{a, b}} \frac{\left|Q h\left(u_{\varphi}(\cdot, s)\right)\right|^{2}}{\left|h\left(Q u_{\varphi}(\cdot, s)\right)\right|} \mathrm{d} s \\
& +\mathbb{E} \int_{0}^{\tau_{a, b}} H^{\prime}\left(Q u_{\varphi}(\cdot, s)\right)\left(Q g\left(u_{\varphi}(\cdot, s)\right)-\frac{g\left(Q u_{\varphi}(\cdot, s)\right)}{\left|h\left(Q u_{\varphi}(\cdot, s)\right)\right|^{2}}\left|Q h\left(u_{\varphi}(\cdot, s)\right)\right|^{2}\right) \mathrm{d} s \\
= & H(Q \varphi)+\mathbb{E} \int_{0}^{t \wedge \tau_{a, b}} \mid Q h\left(u_{\varphi}(\cdot, s) \mid \mathrm{d} s+\mathbb{E} \int_{0}^{t} \Delta(s) \mathrm{d} s\right.
\end{aligned}
$$

with

$$
\begin{aligned}
\Delta(s)= & \frac{\left|Q h\left(u_{\varphi}(\cdot, s)\right)\right|^{2}-\left|h\left(Q u_{\varphi}(\cdot, s)\right)\right|^{2}}{\left|h\left(Q u_{\varphi}(\cdot, s)\right)\right|}+H^{\prime}\left(Q u_{\varphi}(\cdot, s)\right)\left(Q g\left(u_{\varphi}(\cdot, s)\right)-g\left(Q u_{\varphi}(\cdot, s)\right) \frac{\left|Q h\left(u_{\varphi}(\cdot, s)\right)\right|^{2}}{\left|h\left(Q u_{\varphi}(\cdot, s)\right)\right|^{2}}\right) \\
= & H^{\prime}\left(Q u_{\varphi}(\cdot, s)\right)\left(Q g\left(u_{\varphi}(\cdot, s)\right)-g\left(Q u_{\varphi}(\cdot, s)\right)\right) \\
& +\frac{\left|h\left(Q u_{\varphi}(\cdot, s)\right)\right|^{2}-\left|Q h\left(u_{\varphi}(\cdot, s)\right)\right|^{2}}{\left|h\left(Q u_{\varphi}(\cdot, s)\right)\right|}\left(H^{\prime}\left(Q u_{\varphi}(\cdot, s)\right) g\left(Q u_{\varphi}(\cdot, s)\right)-\left|h\left(Q u_{\varphi}(\cdot, s)\right)\right|\right) .
\end{aligned}
$$

Here we are in the frame of (4.8) and (4.9) with the function $H^{\prime}$ instead of $F$. Since $H^{\prime}$ is bounded, we may do the same computation as in the step 1 of the proof of Proposition 4.3 and obtain that

$$
\mathbb{E} \int_{0}^{t \wedge \tau_{a, b}}|\Delta(s)| \mathrm{d} s \leqslant c \mathbb{E} \int_{0}^{t \wedge \tau_{a, b}}\left\|u_{\varphi}(\cdot, s)-Q u_{\varphi}(\cdot, s)\right\|_{2}^{2} \mathrm{~d} s \leqslant c \mathbb{E} \int_{0}^{+\infty}\left\|u_{\varphi}(\cdot, s)-Q u_{\varphi}(\cdot, s)\right\|_{2}^{2} \mathrm{~d} s .
$$

Using (3.5), we deduce that

$$
\lim _{t \rightarrow+\infty} \mathbb{E} \int_{0}^{t \wedge \tau_{a, b}}|\Delta(s)| \mathrm{d} s \leqslant \mathbb{E} \int_{0}^{+\infty}\left\|u_{\varphi}(\cdot, s)-Q u_{\varphi}(\cdot, s)\right\|_{2}^{2} \mathrm{~d} s<+\infty .
$$

Using (4.14) and Fatou's lemma we get

$$
\begin{aligned}
0 & \leqslant \mathbb{E} \liminf _{t \rightarrow+\infty} \int_{0}^{t \wedge \tau_{a, b}}\left|h\left(Q u_{\varphi}(\cdot, s)\right)\right| \mathrm{d} s \\
& \leqslant \liminf _{t \rightarrow+\infty} \mathbb{E} \int_{0}^{t \wedge \tau_{a, b}}\left|h\left(Q u_{\varphi}(\cdot, s)\right)\right| \mathrm{d} s \\
& =\liminf _{t \rightarrow+\infty}\left(-H(Q \varphi)+\mathbb{E} H\left(Q u_{\varphi}\left(\cdot, t \wedge \tau_{a, b}\right)\right)-\mathbb{E} \int_{0}^{t \wedge \tau_{a, b}} \Delta(s) \mathrm{d} s\right)<+\infty
\end{aligned}
$$

thank to (4.15) and the boundedness of $H$. Consequently,

$$
+\infty>\mathbb{E} \liminf _{t \rightarrow+\infty} \int_{0}^{t \wedge \tau_{a, b}}\left|h\left(Q u_{\varphi}(\cdot, s)\right)\right| \mathrm{d} s \geqslant\left(\min _{u \in[a, b]}|h(u)|\right) \mathbb{E} \tau_{a, b}
$$

By hypothesis $(\mathrm{H}), \min _{u \in[a, b]}|h(u)|>0$, then $\mathbb{E} \tau_{a, b}$ is finite for all $a, b \in\left(u_{0}, u_{1}\right)$.

Proof of Theorem 3.4. We put $u_{0}<a<a^{*}<\varphi<b^{*}<b<u_{1}$. It is clear that $\tau_{a^{*}, b^{*}} \leqslant \tau_{a, b} \mathbb{P}$-a.s. We recall that

$$
R(a, b)=\int_{0}^{\tau_{a, b}} \frac{1}{2} F^{\prime \prime}\left(Q u_{\varphi}(\cdot, s)\right)\left|Q h\left(u_{\varphi}(\cdot, s)\right)\right|^{2}+F^{\prime}\left(Q u_{\varphi}(\cdot, s)\right) Q g\left(u_{\varphi}(\cdot, s)\right) \mathrm{d} s .
$$


Following the same arguments we carried out in the proof of Proposition 4.3 step 1 , there exists $\gamma \in(0,1]$ such that

$$
\begin{aligned}
\left|R(a, b)-R\left(a^{*}, b^{*}\right)\right| & \leqslant c \int_{\tau_{a^{*}, b^{*}}}^{\tau_{a, b}}\left\|u_{\varphi}(\cdot, s)-Q u_{\varphi}(\cdot, s)\right\|_{2}^{2 \gamma} \mathrm{d} s \\
& \leqslant c \int_{0}^{+\infty}\left\|u_{\varphi}(\cdot, s)-Q u_{\varphi}(\cdot, s)\right\|_{2}^{2 \gamma} \mathrm{d} s
\end{aligned}
$$

which belongs to $L^{1}(\Omega)$ by $(3.5)$. Moreover,

$$
R(a, b)-R\left(a^{*}, b^{*}\right) \underset{\substack{a, a^{*} \backslash u_{0} \\ b, b^{*} \succ u_{1}}}{\longrightarrow} 0
$$

$\mathbb{P}$-a.s., since the exit times are almost surely finite. By the dominated convergence theorem, we have

$$
\mathbb{E}\left|R(a, b)-R\left(a^{*}, b^{*}\right)\right| \underset{\substack{a, a^{*} u_{0} \\ b, b^{*} u_{1}}}{\longrightarrow} 0 .
$$

Thus there exists a random variable $R \in L^{1}(\Omega)$ such that $R(a, b) \underset{a \searrow_{0}}{\longrightarrow} R$ in $L^{1}(\Omega)$. We take the limit in (4.3) and (4.4) in order to get

$$
\begin{aligned}
\mathbb{P}\left\{\lim _{t \rightarrow+\infty} Q u_{\varphi}(\cdot, t)=u_{0}\right\} & =\lim _{\substack{a u_{0} \\
b \nearrow u_{1}}} \mathbb{P}\left\{Q u_{\varphi}\left(\cdot, \tau_{a, b}\right)=a\right\} \\
& =\frac{F\left(u_{1}\right)-\mathbb{E} F(Q \varphi)}{F\left(u_{1}\right)-F\left(u_{0}\right)}-\frac{\mathbb{E} R}{F\left(u_{1}\right)-F\left(u_{0}\right)} .
\end{aligned}
$$

The above probability is well defined since $F\left(u_{1}\right)$ and $F\left(u_{2}\right)$ are finite in the case (D). We put $Z=\mathbb{E} R$. So $(3.14)$ is proved and similar computations yield (3.15).

\section{StRATONOVITCH'S CASE AND CONCLUDING REMARKS}

We end this work with concluding remarks which allow us to compare our results with those of Chueshov and Vuillermot [6], Chueshov and Vuillermot [7], Bergé et al. [3] and Hetzer et al. [9].

Remark 5.1. In order to compare our results with those of Chueshov and Vuillermot [6], we rewrite the equation (1.1) under the Stratonovitch sense (the "॰" symbol). More precisely, we introduce the following SPDE

$$
\left\{\begin{aligned}
\mathrm{d} u^{S}(x, t)= & \left(\operatorname{div}\left(k(x, t) \nabla u^{S}(x, t)\right)+g^{S}\left(u^{S}(x, t)\right)\right) \mathrm{d} t \\
& +\sum_{j=1}^{r} h_{j}^{S}\left(u^{S}(x, t)\right) \circ \mathrm{d} W_{t}^{j}, \quad(x, t) \in D \times(0,+\infty), \\
u^{S}(x, 0)= & \varphi(x) \in\left(u_{0}, u_{1}\right), \quad x \in D, \\
\frac{\partial u^{S}(x, t)}{\partial n(k)}= & 0, \quad(x, t) \in \partial D \times[0,+\infty),
\end{aligned}\right.
$$

where $g^{S}$ belongs to $\mathcal{C}^{2}\left(\left[u_{0}, u_{1}\right] ; \mathbb{R}\right), h^{S}=\left(h_{1}^{S}, \ldots, h_{r}^{S}\right)$ belongs to $\mathcal{C}^{3}\left(\left[u_{0}, u_{1}\right] ; \mathbb{R}^{r}\right)$ and satisfy $g\left(u_{0}\right)=g\left(u_{1}\right)=$ $h^{S}\left(u_{0}\right)=h^{S}\left(u_{1}\right)=0$. We denote by $\left(u_{\varphi}^{S}(\cdot, t)\right)_{t \in \mathbb{R}^{+}}$the random field solution of SPDE (5.1) starting from $\varphi$. As in Chueshov and Vuillermot [6], we assume that $\left|h^{S}(u)\right|>0$ for all $u \in\left(u_{0}, u_{1}\right),\left|\left(h^{S}\right)^{\prime}\left(u_{0}\right)\right|>0$ and 
$\left|\left(h^{S}\right)^{\prime}\left(u_{1}\right)\right|>0$. Then rewriting the equation of $\left(u_{\varphi}^{S}(\cdot, t)\right)_{t \in \mathbb{R}^{+}}$in Itô's sense yields exactly the SPDE (1.1) with $g=g^{S}+\sum_{j=1}^{r} h_{j}^{S}\left(h_{j}^{S}\right)^{\prime}$ and $h=h^{S}$.

Hence all the results of Chueshov and Vuillermot [6] are included in the results of Section 2 .

Moreover, we can also observe an exchange of stability phenomenon when we write the equation in Stratonovitch's sense (see Sect. 3). This case does not appear in Chueshov and Vuillermot [6] because of their restriction on the sign of the coefficient $g^{S}$.

Remark 5.2. Whereas we recover all the results of Chueshov and Vuillermot [6] in the Stratonovitch case, we did not recover all the results of Bergé et al. [3]. On one hand, if the constant of ellipticity $k_{1}$ is small, this article does not provide the exchange of stability noticed in Bergé et al. [3] when the sign of $g$ is constant. On the other hand, if $k_{1}$ is large enough, we improve their results since we compute the probabilities (3.17) and (3.18). In Bergé et al. [3], the authors were only able to give a lower bound of these probabilities as those given in (3.1) and (3.2). Moreover we have no longer any restriction on the sign of $g$.

Remark 5.3. In the cases (A) and (B), the random field $u_{\varphi}$ has the same asymptotic behaviour as its spatial average, namely

$$
\mathbb{E}\left\|u_{\varphi}(\cdot, t)-Q u_{\varphi}(\cdot, t)\right\|_{2}^{2} \underset{t \rightarrow+\infty}{\longrightarrow} 0
$$

Indeed, in the case $(\mathrm{A})$, we write

$$
\mathbb{E}\left\|u_{\varphi}(\cdot, t)-Q u_{\varphi}(\cdot, t)\right\|_{2}^{2} \leqslant 2 \mathbb{E}\left\|u_{\varphi}(\cdot, t)-u_{0}\right\|_{2}^{2}+2 \mathbb{E}\left\|Q u_{\varphi}(\cdot, t)-u_{0}\right\|_{2}^{2} .
$$

Thanks to (2.9) and the dominated convergence theorem, the first term of the right-hand side of (5.3) tends to 0 . Let us have a closer look at the second one. It is clear that we have almost surely the estimate

$$
u_{0} \leqslant Q u_{\varphi}(\cdot, t) \leqslant v_{2}(t)
$$

Moreover in the case $(\mathrm{A}), v_{2}(t)$ tends to $u_{0}$ almost surely with $t$. So we have (5.2).

Using $v_{1}(t)$ instead of $v_{2}(t)$, the same computations are valid in the case (B) where the roles of $u_{0}$ and $u_{1}$ are inverted. Hence (5.2) is also true in the case (B).

Since the random field $\left(u_{\varphi}(\cdot, t)\right)_{t \in \mathbb{R}^{+}}$converges to its spatial average, it is quite natural to ask if $\left\|\nabla u_{\varphi}(\cdot, t)\right\|_{2}^{2}$ goes to 0 with $t$. In Chueshov and Vuillermot [6], Chueshov and Vuillermot [7] and Bergé et al. [3] the authors gave a result in this way. The following remark specifies the asymptotic behaviour of the gradient of $u_{\varphi}$.

Remark 5.4. We assume that the hypotheses $(\mathrm{K}),(\mathrm{G}),(\mathrm{H})$ and $(\mathrm{I})$ hold. In cases $(\mathrm{C})$ and $(\mathrm{D})$ we moreover assume that the ellipticity constant $k_{1} \geqslant k^{*}$ (see Prop. 3.1). Then we have

$$
\lim _{t \rightarrow \infty} \mathbb{E}\left\|\nabla u_{\varphi}(\cdot, t)\right\|_{2}^{2}=0
$$

Proof. A straightforward adaptation of the proofs of Lemmas 3.9-3.11 of Chueshov and Vuillermot [6] yields that the estimate

$$
\mathbb{E}\left\|\nabla u_{\varphi}(\cdot, t)\right\|_{2}^{2} \leqslant c\left(\mathbb{E}\left\|u_{\varphi}(\cdot, t-2)-Q u_{\varphi}(\cdot, t-2)\right\|_{2}^{2}+\int_{t-2}^{t} \mathbb{E}\left\|u_{\varphi}(\cdot, s)-Q u_{\varphi}(\cdot, s)\right\|_{2}^{2} \mathrm{~d} s\right)
$$

holds for a constant $c>0$ and all sufficiently large $t>0$. Then we use

- the relation (5.2) in cases (A) and (B);

- the exponential decay estimate (3.4) in cases (C) and (D); and the result follows thanks to a dominated convergence argument. 
Remark 5.5. In Hetzer et al. [9], the authors study the asymptotic behaviour of stochastic parabolic equations of Fisher type:

$$
\left\{\begin{array}{l}
\mathrm{d} u=(\Delta u+m(x, u) g(u)) \mathrm{d} t+g(u) \circ \mathrm{d} W_{t}, \quad x \in D, \\
\frac{\partial u}{\partial n}=0, \quad x \in \partial D .
\end{array}\right.
$$

There are not many differences between the two formulations: on one hand, our evolution operator is more general than in the Laplacian operator and on the other hand, their drift coefficients may depend on the space variable.

The authors also proved that three alternatives are met. The two first ones are the convergence towards one of the trivial equilibrium, or oscillation between them. The last alternative says that every solution is neither bounded away from the trivial equilibrium nor converges to them. This result is essentially contained in Theorem 2.17 and in the estimations (3.1) and (3.2) appearing in our case (D). Moreover, in our work, we give a more precise description of this asymptotic behaviour in terms of the attraction towards a Bernoulli law.

Acknowledgements. We are grateful to an anonymous referee who inform us about the existence of Hetzer et al.'s article [9].

\section{REFERENCES}

[1] L. Arnold, Stochastic Differential Equations: Theory and Applications. John Wiley and Sons, New York (1974).

[2] D.G. Aronson and H.F. Weinberger, Nonlinear dynamics in population genetics, combustion and nerve pulse propagation. Lect. Notes Math. 446 (1975) 5-49.

[3] B. Bergé, I.D. Chueshov and P.A. Vuillermot, On the behavior of solutions to certain parabolic SPDE's driven by Wiener processes. Stoch. Proc. Appl. 92 (2001) 237-263.

[4] H. Brézis, Analyse fonctionnelle, théorie et applications. Masson, Paris (1993).

[5] I.D. Chueshov, Monotone Random Systems: Theory and Applications. Lect. Notes Math., Springer, Berlin 1779 (2002).

[6] I.D. Chueshov and P.A. Vuillermot, Long-time behavior of solutions to a class of stochastic parabolic equations with homogeneous white noise: Stratonovitch's case. Probab. Theory Relat. Fields 112 (1998) 149-202.

[7] I.D. Chueshov and P.A. Vuillermot, Long-time behavior of solutions to a class of stochastic parabolic equations with homogeneous white noise: Itô's case. Stochastic Anal. Appl. 18 (2000) 581-615.

[8] I.I. Gihman and A.V. Skorohod, Stochastic Differential Equations. Ergebnisse der Mathematik und ihrer Grenzgebiete, Bd. 72. Springer, Berlin (1972).

[9] G. Hetzer, W. Shen and S. Zhu, Asymptotic behavior of positive solutions of random and stochastic parabolic equations of fisher and Kolmogorov type. J. Dyn. Diff. Eqs. 14 (2002) 139-188.

[10] R.Z. Hasminskii, Stochastic Stability of Differentiel Equations. Alphen, Sijthoff and Nordhof (1980).

[11] N. Ikeda and S. Watanabe, Stochastic Differential Equations and Diffusion Processes, North-Holland Mathematical Library. North-Holland, Kodansha 24 (1981).

[12] A. Kolmogoroff, I. Petrovsky and N. Piscounoff, Étude de l'équation de la diffusion avec croissance de la quantité de matière et son application à un problème biologique. Bull. de l'Univ. d'État à Moscou, série internationale 1 (1937) 1-25.

[13] R. Manthey and K. Mittmann, On a class of stochastic functionnal-differential equations arising in population dynamics. Stoc. Stoc. Rep. 64 (1998) 75-115.

[14] J.D. Murray, Mathematical Biology. Second Edition. Springer, Berlin 19 (1993).

[15] B. Øksendal, G. Våge and H.Z. Zhao, Asymptotic properties of the solutions to stochastic KPP equations. Proc. Roy. Soc. Edinburgh 130A (2000) 1363-1381.

[16] B. Øksendal, G. Våge and H.Z. Zhao, Two properties of stochastic KPP equations: ergodicity and pathwise property. Nonlinearity 14 (2001) 639-662.

[17] M. Sanz-Solé and P.A. Vuillermot, Equivalence and Hölder-Sobolev regularity of solutions for a class of non-autonomous stochastic partial differential equations. Ann. Inst. H. Poincaré Probab. Statist. 39 (2003) 703-742. 